\title{
The Habitat Structure of Lukanga Ramsar Site in Central Zambia: An Understanding of Wetland Ecological Condition
}

\author{
Harry Chabwela ${ }^{1}$, Chansa Chomba ${ }^{2 *}$, Loyd Thole ${ }^{3}$ \\ ${ }^{1}$ Department of Biological Sciences, University of Zambia, Lusaka, Zambia \\ ${ }^{2}$ School of Agriculture and Natural Resources, Disaster Management Training Centre, Mulungushi University, Kabwe, Zambia \\ ${ }^{3}$ Zambia Environmental Management Authority, Ministry of Lands and Natural Resources, Lusaka, Zambia \\ Email:*chansachomba@rocketmail.com,cchomba@mu.ac.zm,ritachansa@yahoo.com
}

How to cite this paper: Chabwela, H., Chomba, C. and Thole, L. (2017) The Habitat Structure of Lukanga Ramsar Site in Central Zambia: An Understanding of Wetland Ecological Condition. Open Journal of Ecology, 7, 406-433.

https://doi.org/10.4236/oje.2017.76029

Received: April 4, 2017

Accepted: June 26, 2017

Published: June 29, 2017

Copyright $\odot 2017$ by authors and Scientific Research Publishing Inc. This work is licensed under the Creative Commons Attribution International License (CC BY 4.0).

http://creativecommons.org/licenses/by/4.0/

\section{(c) (i) Open Access}

\begin{abstract}
A field survey was carried out to determine the vegetation structure of the Lukanga Swamp Ramsar site in central Zambia. The aim of the study was to identify the different vegetation communities, species composition and distribution patterns for improved habitat management. Sampling was conducted in all recognizable vegetation communities. The results of the survey showed that the swamp was a littoral palustrine wetland predominantly characterized by the dominance of Leersia hexandria Swartz (42.02\%), Typha capensis Rohrb (62.43\%), Phragmites australis (Cav.) Trin. ex Steud (33.61\%), Aeschynomeme fluitans Peter (31.58\%) and Polygonum senegalense Meisn (48.8\%). The occurrence of Vossia cuspidata Griff and Cyperus papyrusL was restricted to small and isolated locations. Short Termitaria was generally covered by Acrocerus macrum Stapf (35.25\%) while tall Termitaria was dominated by Panicum maximum Jacq. (26.00\%). The most important woody plant species included Combretum ghasalense Engl. et Diels (I. V = 62.88), Pseudolachnostylis maprouneifolia Pax ((I. V = 90.48), Albizia adianthifolia (Schmacher) W.F. Wight (I. V = 135.63) Isoberlinia angolensis Hyle and Brenan (I. $\mathrm{V}=87.25)$. The current structure of the hydrophytes composition observed in this study was an indication of a generally silting wetland, while the dominant occurrence of understorey woody plants in the surrounding vegetation signified degraded miombo vegetation. It was concluded that the ecological status of wetland habitat was potentially threatened by mainly anthropogenic activities such as; wildfires, and unsustainable exploitation of surrounding vegetation. Further research is required to examine water flows, eutrophication and the long-term effects of deforestation on the ecological functioning of the wetland.
\end{abstract}




\section{Keywords}

Aquatic Macrophytes, Wetland Habitat, Brachystegia Woodland, Palustrine Swamp, Sedimentation, Environmental Flows, Wetland Fires and Overexploitation

\section{Introduction}

The Ramsar Convention, defines Wetlands as "areas of marsh, fen, peat land, or water, whether natural or artificial, permanent or temporary with water that is static or flowing, fresh, brackish or salty, including areas of marine water the depth of which at low tide does not exceed six meters" [1]. They are among the most ecologically productive ecosystems but have in the last few decades suffered severe degradation due to increased demand for their resources by humans.

In Zambia wetlands of three categories exist viz, 1) Riverine system, 2) Lacustrine system, and the Palustrine system occupying nearly $20 \%$ of the land [2] [3] [4]. As transitional zones, wetlands accommodate a myriad of anthropogenic activities. Lukanga Swamp Ramsar site, for instance, recorded anthropogenic activities include; fishing, trophy and meat hunting, livestock grazing, firewood collection and charcoal production, agriculture and water supply for commercial and domestic needs (Figures 1(a)-(d)). Wetlands also generally, influence human settlement patterns as most of them are established within or near lakes and dambos and along rivers and streams in Zambia. Despite their values, no detailed study of the structure of vegetation communities has been carried out in Zambia. Consequently, most wetlands have degraded and species depending on them equally threatened. Over exploitation through overfishing, illegal hunting of large mammals as well as increasing levels of deforestation remains a major concern of nearly all wetlands in the country.

The Lukanga Swamp was specifically selected for this study due to its proximity to the Kafue National Park ecosystem and consequently considered to be part of the Kafue National Park wildlife habitat system. It also serves as an important source of income for people in the central part of Zambia as well as the Copperbelt and Lusaka areas which are also the most urbanized parts of the country. It was as also declared as a Ramsar site in 2006, signifying its importance at national and international levels.

The main goal for carrying out this study was to assess and classify the wetland ecological status [5] [6] and character as a means of stimulating wetland research in the country and elsewhere in the region; and to ensure effective management, planning and monitoring. To achieve this goal, we used vegetation and in particular plant species occurrence and distribution to describe the wetland's ecological status, a method which has been used for similar studies in other wetlands [7] [8]. Research findings in this study were expected to be of great value to the conservation fraternity as a contribution to conservation and 


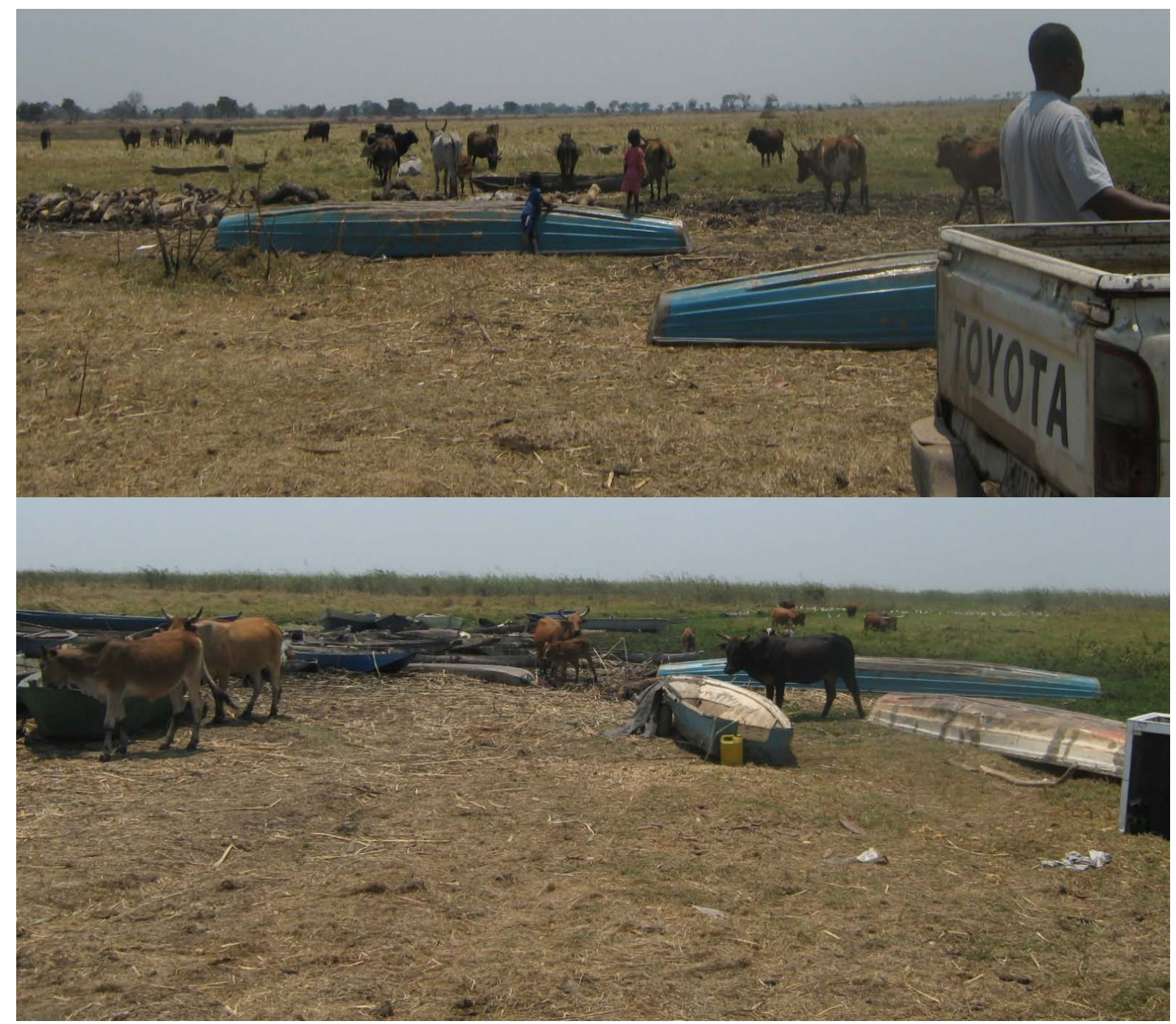

(a)

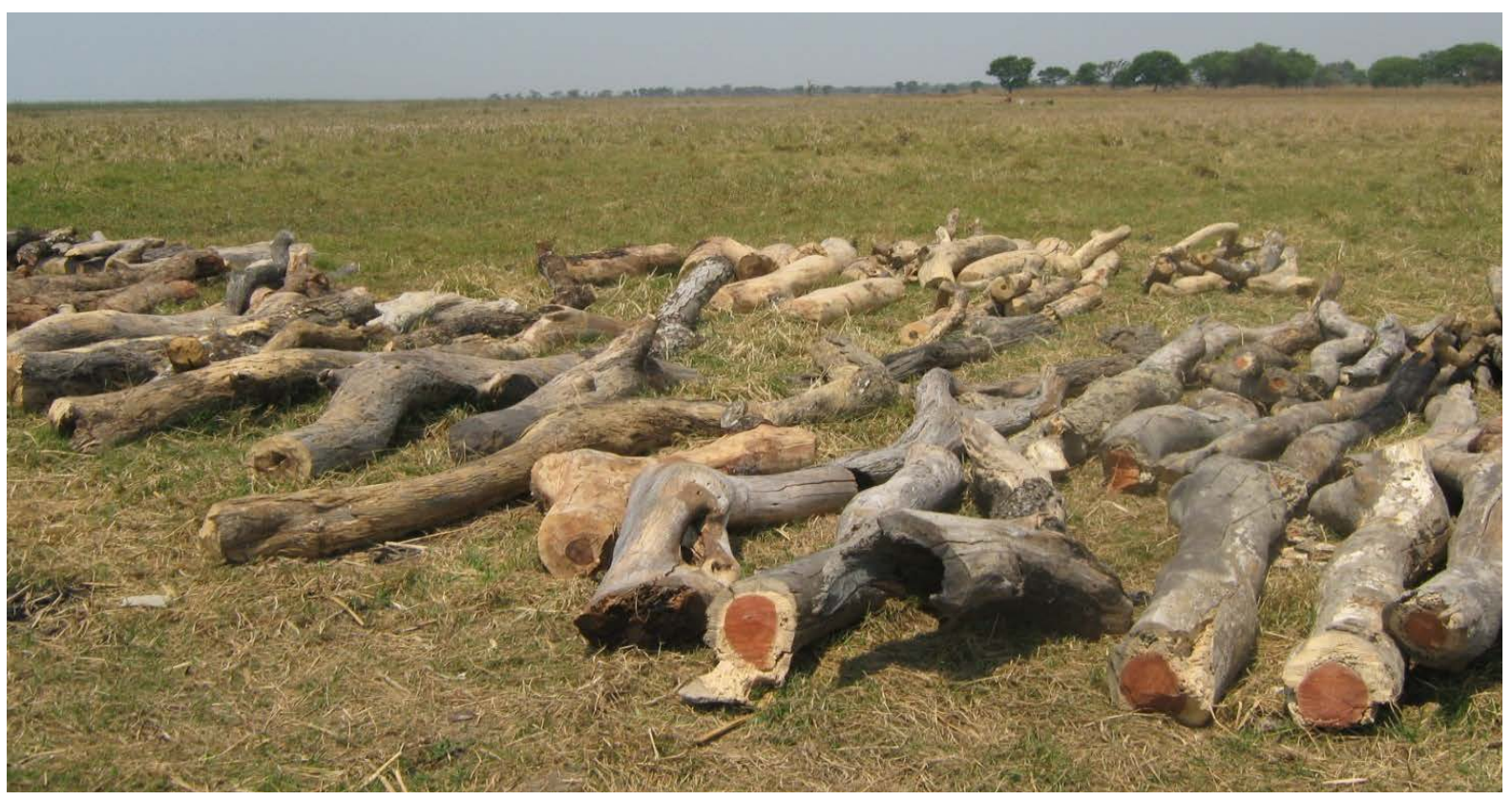

(b) 


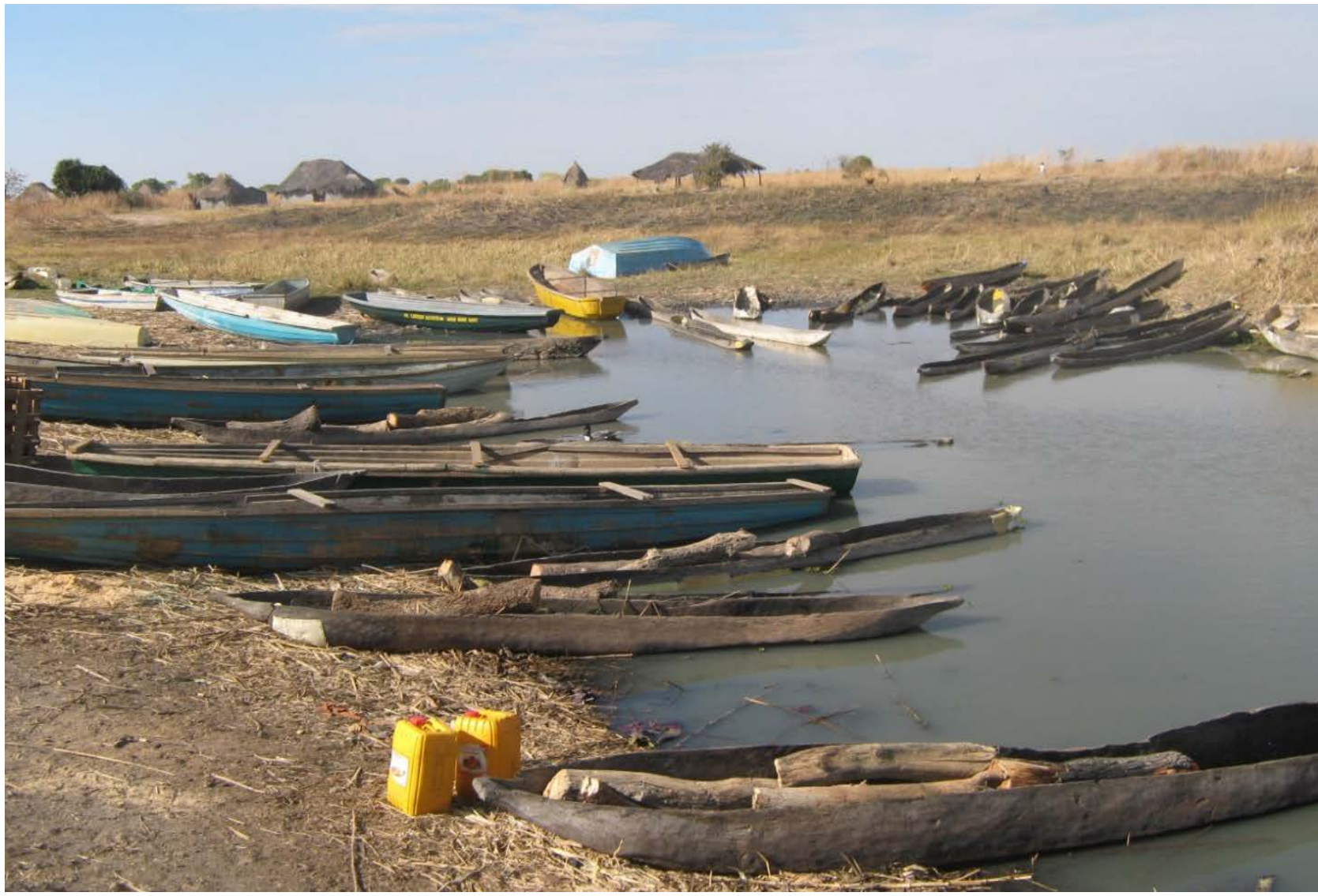

(c)

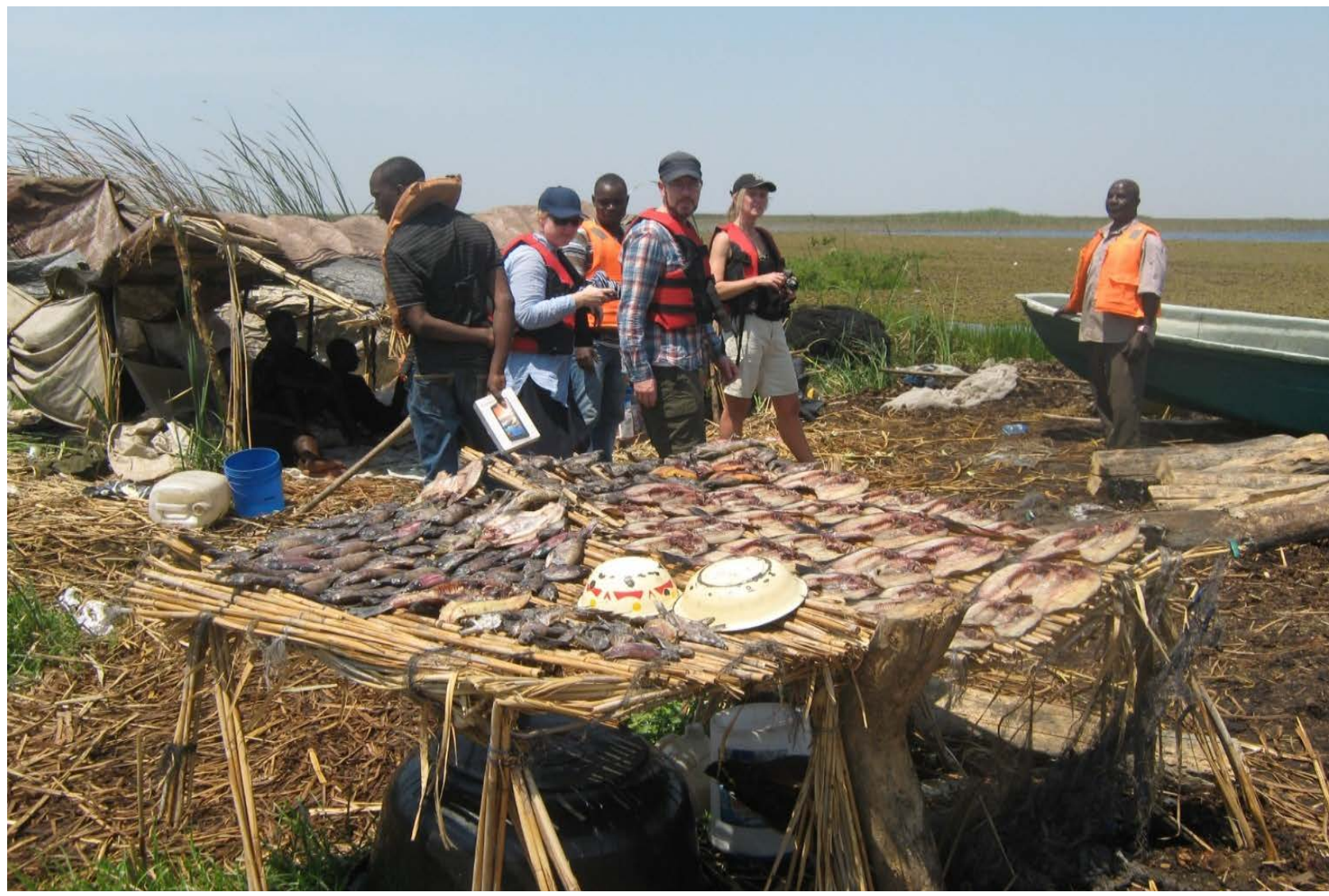




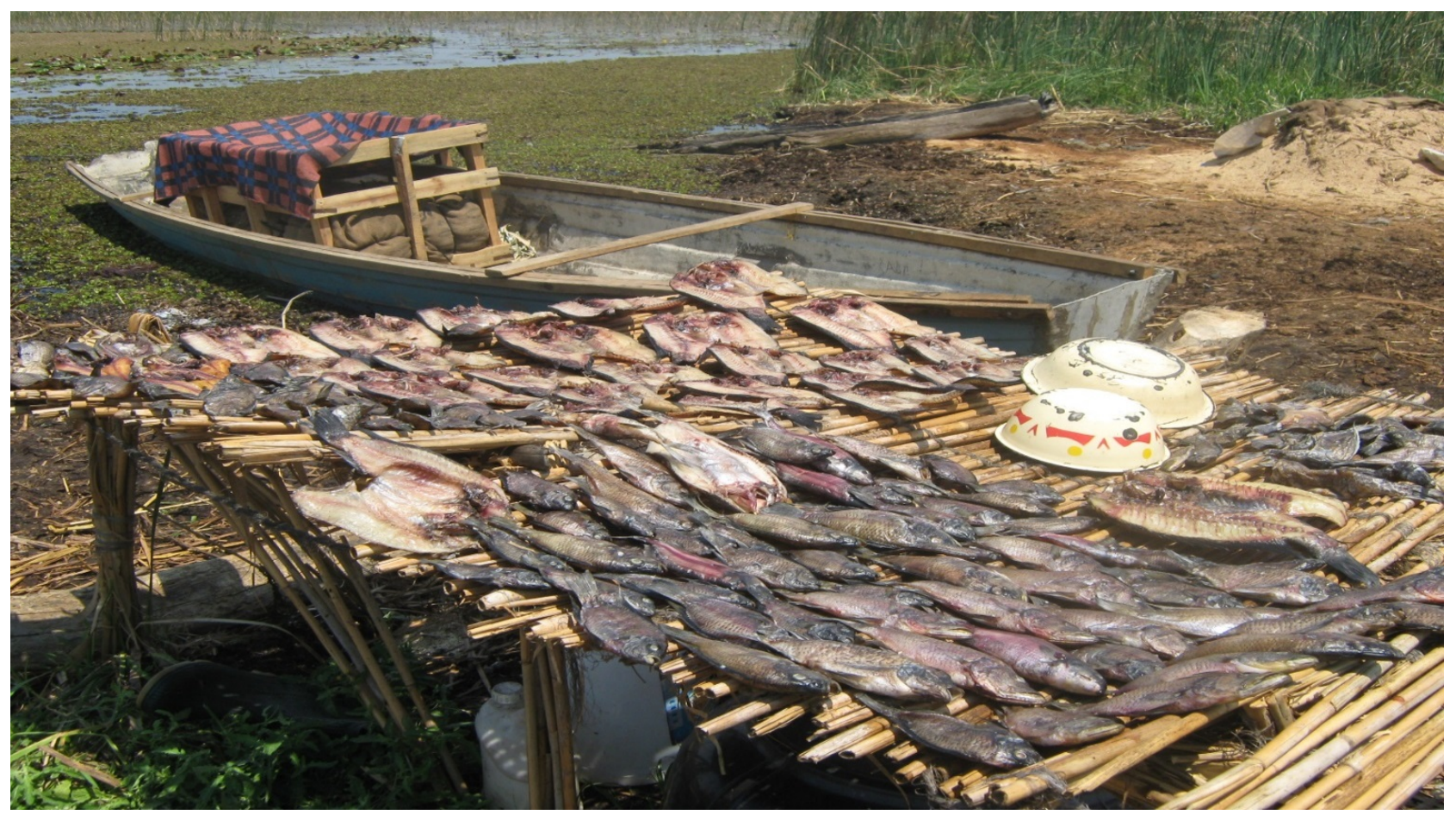

(d)

Figure 1. Human activities in the Lukanga Ramsar site: (a) Cattle grazing and boats used for fishing and transportation; (b) Fire wood cut from the surrounding woody vegetation for drying of fish; (c) Dugout canoes and banana boats at Waya harbor; (d) Drying of fish to sale to urban areas.

management of this wetland whose future critically lies in the available ecological knowledge and information.

\section{Methods and Materials}

\subsection{Study Area Description}

The Lukanga Swamp is a shallow wetland with depth not exceeding $6.1 \mathrm{~m}$, even at the height of the wet season. The area of permanent swamp and open water is approximately $2100 \mathrm{~km}^{2}$, comprising three characteristic types; palustrine, lacustrine and riverine. The palustrine wetland covers approximately $95 \%$ of the area and includes permanent swamp, termitaria grasslands and dambos. The lacustrine wetlands cover about $5 \%$ of the wetland. The riverine wetlands occur in small areas along the fringes of the Lukanga and Mushingashi rivers. Water in the swamp comes from three sources; direct rainfall into the swamp, inflow from tributary streams and spill from the Kafue River. The swamps significantly influence the flows into the Itezhi-tezhi reservoir, located downstream on the Kafue River. The main river flowing into the swamp is the Lukanga, which originates north of the wetland in the Copperbelt Province.

\subsubsection{Location}

The Lukanga Swamp is located approximately $50 \mathrm{~km}$ west of the city of Kabwe, in the Central Province of Zambia. It is strategically positioned within the catchment of the Kafue River [9]. The general elevation is between $1250 \mathrm{~m}$ on the 
catchment area and $1100 \mathrm{~m}$ in the main swamp. The total catchment is 19,490 $\mathrm{km}^{2}$, but the main swamp area is only $2500 \mathrm{~km}^{2}$, extending to $2600 \mathrm{~km}^{2}$ at high floods. This study covered the main swamp and islands, and part of the catchment area along the Kafue River between Nkomonshya Ferry and Mongo at the Lukanga-Kafue River confluence (Figure 2).

\subsubsection{Geology and Soils}

The Lukanga Swamp region is generally covered by rock formations of the Pre-Cambrian assembly of the Gondwana super continent which included the southern continents with series of events occurring between 3000 million years to 100 million years [10]. This rock formation covers much of the eastern side of the Lukanga Swamp. Much of this includes the Katanga system which has wide occurrence in Zambia, and it is the most dominant rock formation in the Lukanga area. The second rock formation bear its origin from late Mesozoic phase which includes the present-day era, covering the periods of the Cretaceous through Tertiary to Quaternary [11] [12]. In Zambia, the late Tertiary period is known as the Kalahari system whose deposits consists of poorly consolidated sand stones, and unconsolidated windblown deposits [12] and much of the western part of the Lukanga Swamp and Chilwa Island is underlain by the Kalahari sands.

Soil types were earlier described [13] as comprising seven types based on UNFAO classification. However, the most widespread soil types in this region include the swamp soils which are organic soils and have very high content of vegetative matter, while the sand veldt soils are loamy sands, coarse grained, but with reasonable content of clay. The sandy soils are generally yellowish-red to light yellowish-brown where well drained, but these change to grey-brown where poorly drained.

\subsubsection{Climate}

Three distinct seasons are recognized viz, 1) rainy/wet season extending from November to March, 2) cool dry season from April to August, and 3) hot, dry season from September to October/November [11] [12]. Mean maximum temperatures are highest in October $\left(31.8^{\circ} \mathrm{C}\right)$ while mean minimum temperature is $14.8^{\circ} \mathrm{C}$. In general, significant amounts of rainfall start in November and continue until early April. However, most of the rains occur mainly in the months of December (243 mm), January (257 mm), February $(190 \mathrm{~mm})$ and March $(126$ $\mathrm{mm}$ ). The number of rainy days for the rainfall of at least $1 \mathrm{~mm}$ is on the average higher in December (18 days) followed by January (16 days) and February (14 days). This trend is the same for the number of rainy days for the rainfall higher than $10 \mathrm{~mm}$. In some years, very low rainfall has been recorded and this has contributed significantly to the drying up of the Lukanga Swamp as was the case between 1986 and 1995 seasons [3].

\subsubsection{Drainage and Hydrology}

The hydrology of the Lukanga Swamp has been fairly described [14] [15] [16] [17] [18]. It is generally shallow with an average depth of $1.5 \mathrm{~m}$, although it can 


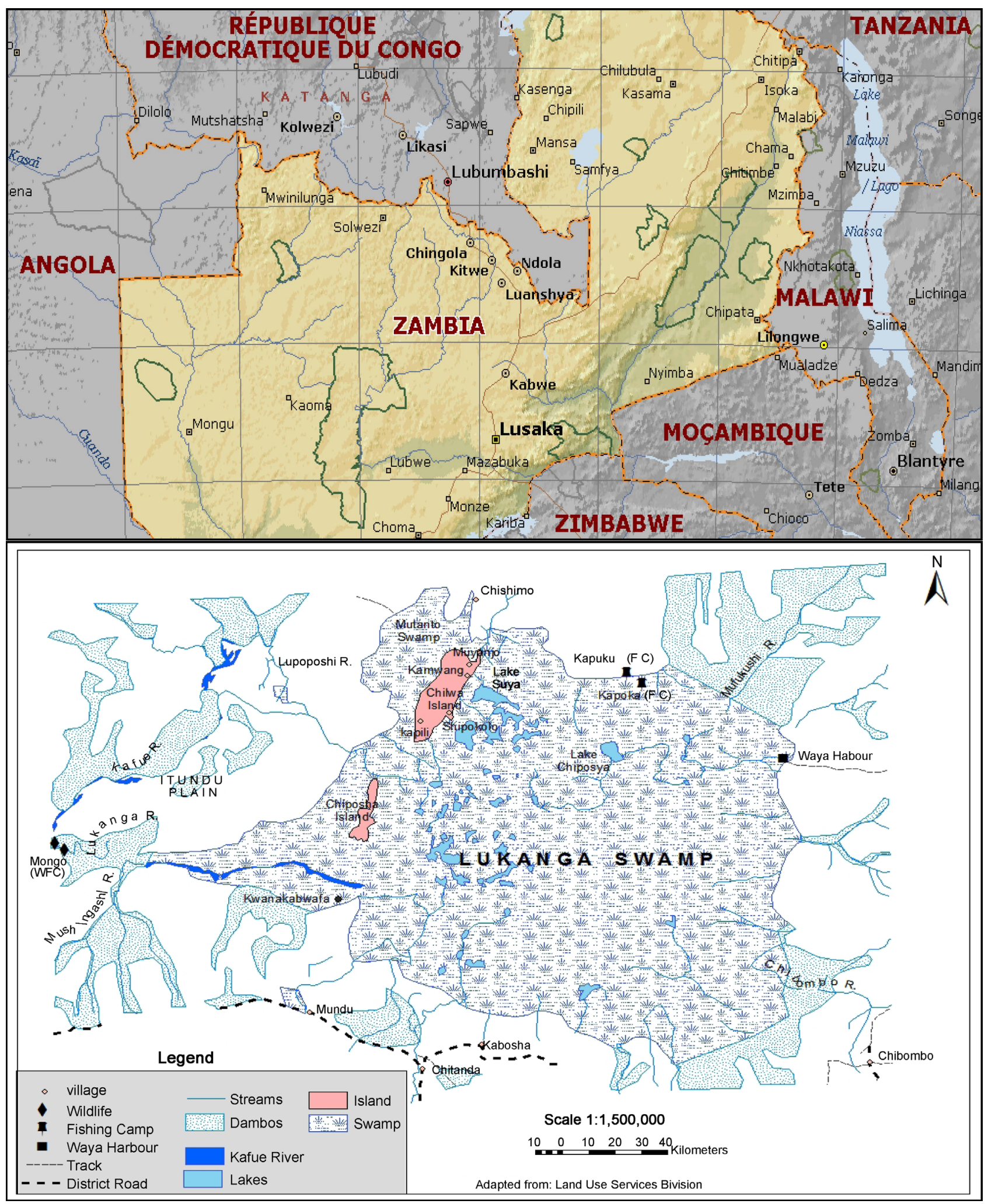

Figure 2. Location of Lukanga swamp Ramsar site in Zambia, 2017.

exceed $6.0 \mathrm{~m}$ in some areas or in exceptionally high floods. The capacity of the swamp depression between the average low water level and the maximum level is approximately $13,000 \mathrm{~m} \mathrm{~m}^{3}$ [16]. The main sources of water are rainfall, surface 
run off, sub surface and Lukanga River and other channels which drain into the swamp from the catchment area. Further inflow comes from the Kafue River through Mwinuna channel. The flows from the streams are seasonal, and cease at the end of the rainy season. Inflows from the Kafue River become possible once the channel flow is above the swamp through the Mwinuna channel via Mutanta Swamp and the southern part of Chilwa Island (see Figure 2). Further inflows from the Kafue River spill over of the banks during very high floods. Conversely, the wetland is drained by the Lukanga River through the Mukunkwa channel and by the back flow through Mwinuna channel, which takes place when water levels in the swamp are higher than that of the Kafue River [15] [16].

\subsection{Data Collection Techniques}

\subsubsection{Vegetation Surveys}

Field surveys involved the use of maps of 1:250,000 and 1: 50,000 to design transect surveys and to carry out verification in two (2) woodland vegetation and seven (7) main swamp vegetation communities. In main swamp where it was not possible to walk on foot (Figure 3), transects were traversed by canoe and in woodlands on foot. Plant species identification was achieved by the use of field guide books [19] and samples of plants which were difficult to identify in the field were taken to the herbarium at the University of Zambia for identification.

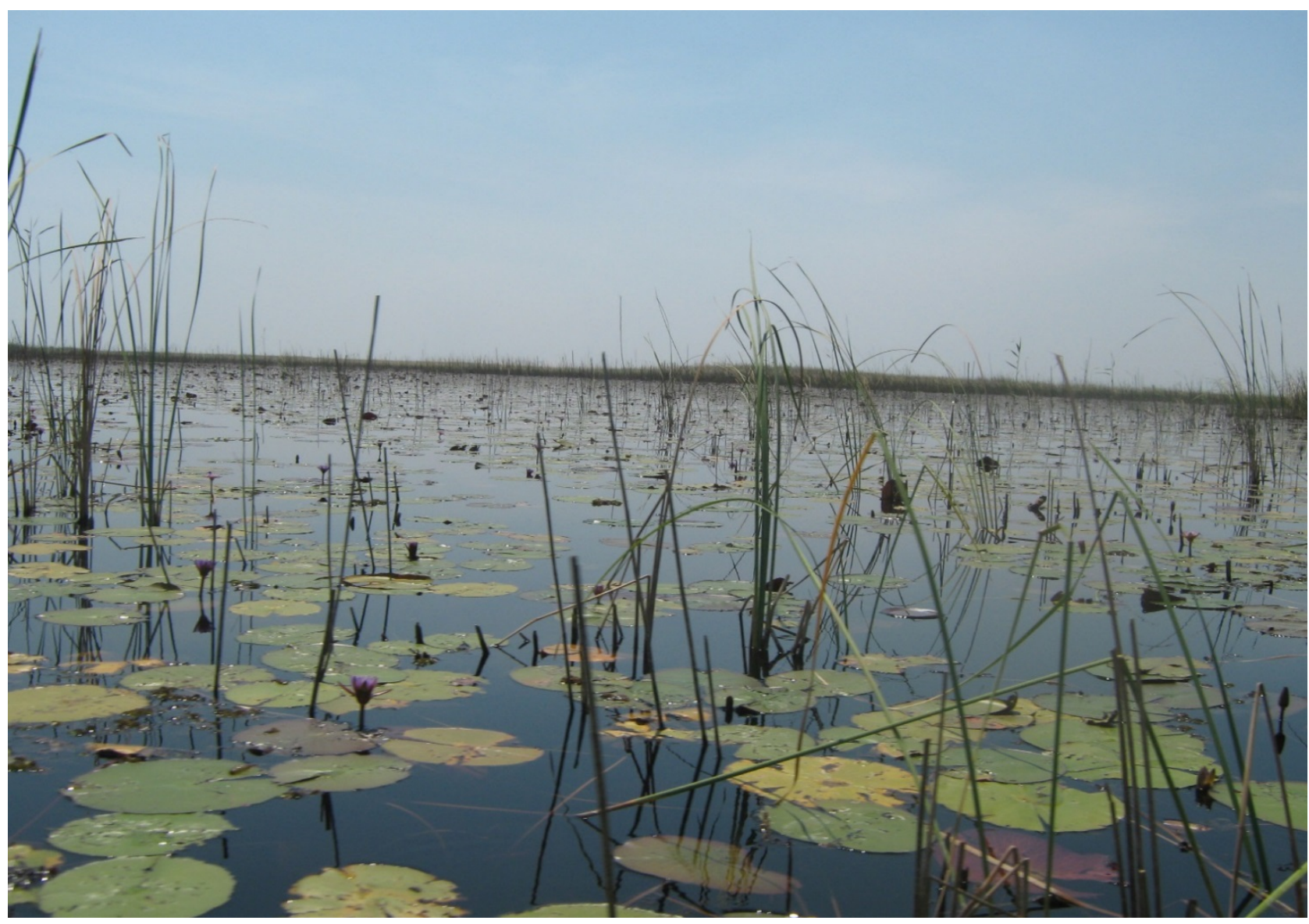

Figure 3. Main swamp where transects were traversed by boat, Lukanga Swamp, Zambia. 


\subsubsection{Herbaceous Pant Assessment}

Data collection on plant species composition was done in six habitat types between Waya and Chilwa Islands (see Figure 2). Plant composition was then estimated by using a $1 / 2$ square meter frame [20]. Each $1 / 2$ square meter frame was subdivided into four (4) equal plots. A total of 320 plots were equally distributed at $10 \mathrm{~m}$ apart along a canoe transect in the permanent swamp (see Figure 3), and at $50 \mathrm{~m}$ apart along a compass line in the tall and short Termitaria habitats. At every $10 \mathrm{~m}$ interval, the quarter square meter frame was lowered on the side of the observer sitting in a canoe; while in the Termitaria, the half square meter frame was lowered in front of the observer. Plant species present in the plot were tallied within each subplot and qualitatively assigned dominance status of the area covered by each plant. Thus, only a dominant plant was recorded in each subplot.

\subsubsection{Woody Plants Assessment}

We sampled five (5) sites in the woodland vegetation: Acacia-Combretum Woodland at the side of Waya, and in the Brachystegia woodland at the Southern part of Waya, Chilwa Island North, Chilwa Island South and Maunde Forest in the North West of the swamp (see Figure 2). Woody vegetation structure was measured using the Point-Centred Quarter (PCQ) method [21] [22] [23]. This required establishing plots at 50 meter intervals along a compass line in each community category. A total of 30 stations in each sample area were established and diameter at breast height (DBH) (at 1.3 meters above ground) of trees occurring in each plot was taken by using a cruising stick and a measuring tape.

Relative dominance, relative density, relative frequency and importance value were analyzed as shown below;

$$
\begin{gathered}
\text { Relative Dominance }=\frac{\text { Dominance of species }}{\text { Total dominance of all species }} \times 100 \\
\text { Relative Density }=\frac{\text { Number of individuals of a species }}{\text { Total number of individuals (all species) }} \times 100 \\
\text { Relative Frequency }=\frac{\text { Frequency of a species }}{\text { Sum frequency of all species }} \times 100
\end{gathered}
$$

Importance Value $=$ Relative density + Relative dominance + Relative frequency $(\mathrm{d})$

\section{Results}

\subsection{Habitat Types}

Eight (8) habitat types were recognized, miombo woodland, Acacia-Combretum woodland, Baikea woodland, Kalahari, Lake basin Chipya, termitaria grassland, permanent swamp and open water (Figure 4).

\subsubsection{Aquatic Macrophytes Community Structure}

The distribution of aquatic macrophytes in six (6) plant communities of the swamp wetland is presented in Table 1. 


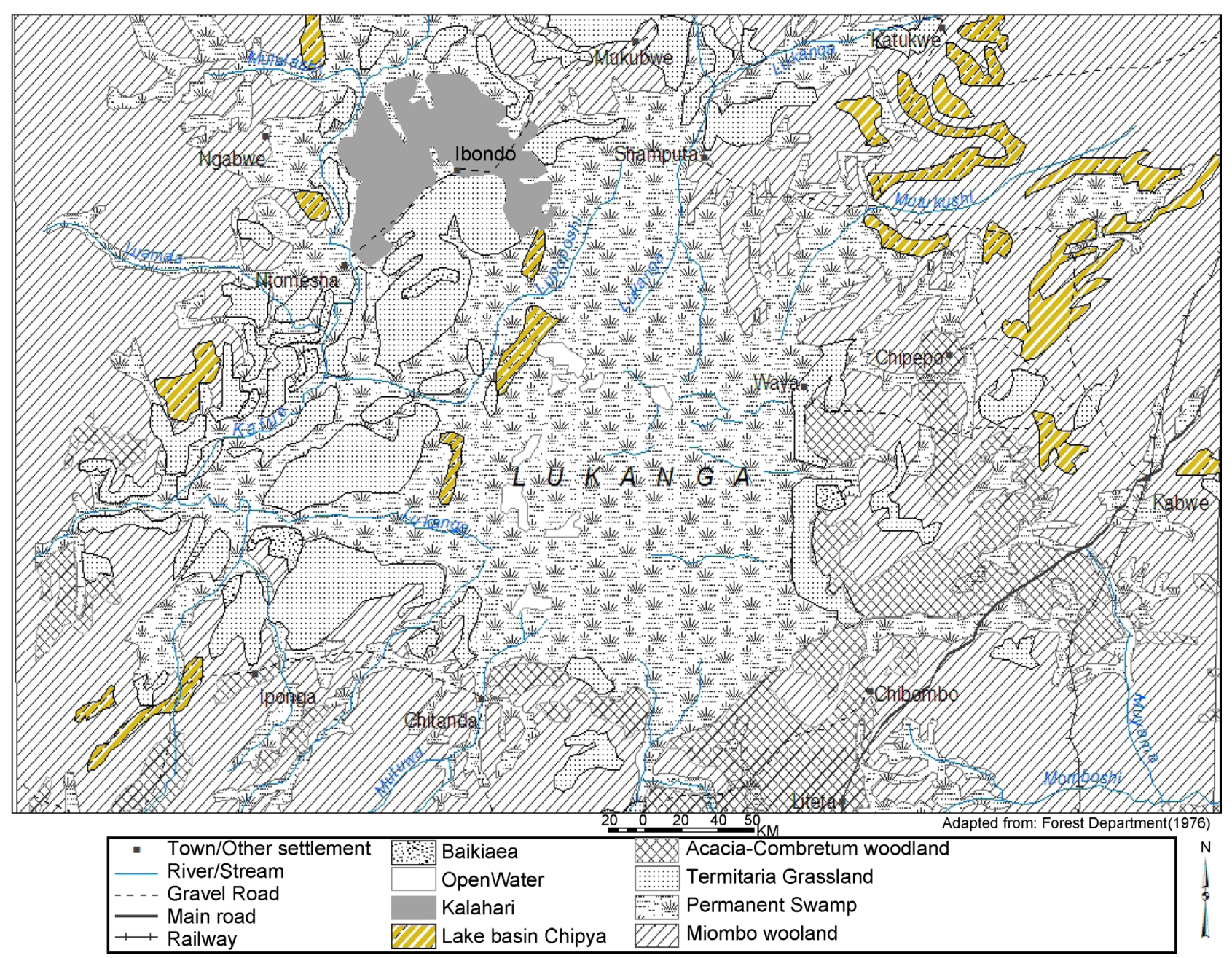

Figure 4. Spatial distribution of eight habitat types was recognized during the study, Lukanga Swamp, Zambia.

\subsubsection{Phragmites australis Community}

Based on the percent occurrence of dominant plant species, the dry reed bed zone had Leersia hexandra Swartz (42.02\%), Phragmites australis (Cav.) and Trin. ex Steud (33.61\%) as co-dominants (Figure 5). However, species such as Aeschynomene fluitans Peter (12.6\%), Ipomea aquatic Forsk (6.51\%), Ludiwigia leptocarpa (Nutt) Hara (5.25\%), Typha capensis Rohrb (2.5\%) and Vossia cuspidata Griff (2.5\%) were equally important (Table 1). Other species with less than two percent representation included Nymphaea capensis Thunb, Eleocharis dulcis (burm.f) Trin ex Herschel, Polygonum senegalinse Meisn, Scirpus sp. and Utricularia L.

\subsubsection{Nymphaea capensis Community}

This plant community was significant in the vegetated areas of the shallow parts of the lakes, and the proportions of common plants species are given in Table 1. Nymphaea capensis Thunb (35.40\%), Typha capensis Rohrb (25.39\%), Phragmites australis (Cav.) Trin. ex Steud (13.36\%), Polygonum senegalinse Meisn (8.71\%), Ipomea aquatic Forsk (8.60\%), Eleocharis dulcis (burm.f) Trin ex Herschel (5.73\%), Leersia hexandra Swartz (3.8\%), Cyperus papyrus L (2.5\%), 
Table 1. Species composition of emergent macrophytes vegetation based on relative frequency in five plant communities.

\begin{tabular}{|c|c|c|c|c|c|c|c|}
\hline \multicolumn{8}{|c|}{ PLANT COMMUNITIES } \\
\hline SPECIES & $\begin{array}{c}\text { Tall } \\
\text { Termitaria }\end{array}$ & $\begin{array}{c}\text { Short } \\
\text { Termitaria }\end{array}$ & $\begin{array}{c}\text { Phragmites } \\
\text { australis }\end{array}$ & $\begin{array}{l}\text { Nymphaea } \\
\text { capensis }\end{array}$ & $\begin{array}{l}\text { Polyganum } \\
\text { senegalinse }\end{array}$ & $\begin{array}{l}\text { Typha } \\
\text { capensis }\end{array}$ & $\begin{array}{c}\text { Aeschnomene } \\
\text { fluitans }\end{array}$ \\
\hline Acrocerus macrum & 0.0 & 35.25 & 0.0 & 0.0 & 0.0 & 0.0 & 10.35 \\
\hline Aeschynomene fluitans & 0.0 & 22.52 & 12.60 & 0.0 & 0.0 & 0.0 & 31.58 \\
\hline Brachiaria brizatha & 10.92 & 0.0 & 0.0 & 0.0 & 0.0 & 0.0 & 0.0 \\
\hline Cymbopogon valida & 13.00 & 0.0 & 0.0 & 0.0 & 0.0 & 0.0 & 0.0 \\
\hline Cyperus papyrus & $0, .0$ & 0.0 & 0.0 & 2.5 & 0.0 & 0.0 & 0.0 \\
\hline Digitaria milanjiana & 15.25 & 0.0 & 0.0 & 0.0 & 0.0 & 0.0 & 0.0 \\
\hline Eleocharis dulcis & 0.0 & 0.0 & 0.0 & 5.73 & 7.5 & 22.35 & 0.0 \\
\hline Elodea sp. & 0.0 & 0.0 & 0.0 & 2.5 & 0.0 & 0.0 & 0.0 \\
\hline Hibiscus spp. & 10.92 & 0.0 & 0.0 & 0.0 & 0.0 & 0.0 & 0.0 \\
\hline Imperata cylindrica & 0.0 & 0.0 & 0.0 & 0.0 & 0.0 & 0.0 & 5.26 \\
\hline Ipomea aquatic $\mathrm{F}$ & 0.0 & 22.52 & 6.51 & 8.60 & 0.0 & 0.0 & 0.0 \\
\hline Leersia hexandra & 0.0 & 0.0 & 42.02 & 0.0 & 0.0 & 0.0 & 36.84 \\
\hline Ludiwigia leptocarpa & 0.0 & 0.0 & 5.25 & 0.0 & 0.0 & 0.0 & 0.0 \\
\hline Nymphaea capensis & 0.0 & 0.0 & 0.0 & 35.40 & 26.3 & 0.0 & 0.0 \\
\hline Panicum maximum & 26.00 & 0.0 & 0.0 & 0.0 & 0.0 & 0.0 & 0.0 \\
\hline Phragmites australis & 0.0 & 14.10 & 33.61 & 13.36 & 5.0 & 15.22 & 0.0 \\
\hline Polyganum senegalinse & 0.0 & 0.0 & 0.0 & 8.71 & 48.8 & 0.0 & 0.0 \\
\hline Scirpus spp. & 0.0 & 0.0 & 0.0 & 2.5 & 0.0 & 0.0 & 0.0 \\
\hline Sesbania rostrata Bremek & 0.0 & 5.63 & 5.63 & 0.0 & 0.0 & 0.0 & 0.0 \\
\hline Setaria ancept & 13.00 & 0.0 & 0.0 & 0.0 & 0.0 & 0.0 & 0.0 \\
\hline Sorghum verticilliflorum & 10.92 & 0.0 & 0.0 & 0.0 & 0.0 & 0.0 & 0.0 \\
\hline Typha capensis & 0.0 & 0.0 & 0.0 & 27.39 & 12.5 & 62.43 & 0.0 \\
\hline Utricularia spp & 0.0 & 0.0 & 0.0 & 2.5 & 0.0 & 0.0 & 0.0 \\
\hline Vossia cuspiidata & 0.0 & 0.0 & 0.0 & 0.0 & 0.0 & 0.0 & 15.79 \\
\hline Total & 100 & 100 & 100 & 100 & 100 & 100 & 100 \\
\hline
\end{tabular}

Utricularia spp. (2.5\%) and Scirpus spp. (1.2\%) showed great prevalence in this community.

\subsubsection{Polygonum senegalinse Community}

In general, this community had a low number of plant species. As shown in Table 1, the habitat was dominated by Polygonum senegalense Meisn (48.8\%), Nymphaea capensis Thunb (26.3\%), Typha capensis Rohrb (12.5\%), Eleocharis dulcis (burm.f) Trin ex Herschel (7.5\%) and Phragmites australis (Cav.) Trin. ex Steud (5\%).

\subsubsection{Typha capensis Community}

This was the second largest swamp plant community and dominated deeper water areas, replacing Phragmites community. As shown in Table 1 (also see Figure 3) this plant community was largely represented by Typha capensis Rohrb 


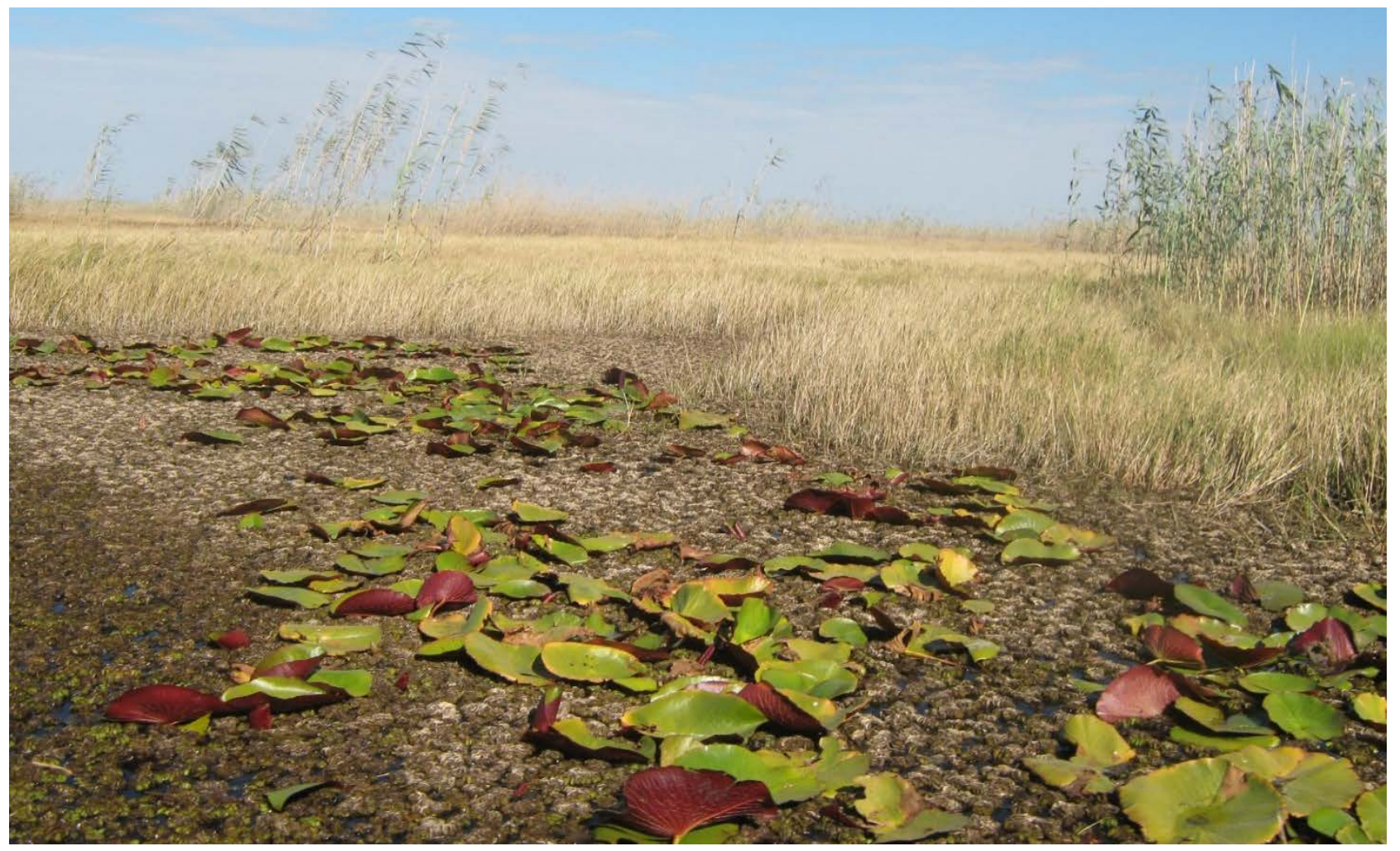

Figure 5. Phragmites australis top right corner and, Lukanga Swamp, Zambia.

(62.43\%) followed by Phragmites australis (Cav.) Trin. ex Steud (15.22\%) and Eleocharis dulcis (burm.f) Trin ex Herschel (22.35\%). However, there were also some emergent macrophytes occurring in low proportions including Nymphaea capensis Thunb., Polygonum senegalinse Meisn., Ceratophyllum demersum L. and Leersia hexandra Swartz.

\subsubsection{Aeschynomene fluitans Community}

This plant community was common at the mouths of Lukanga and Lupoposhi rivers, and it also occupied edges of permanently flooded areas, usually preferring areas that were covered with water. Common plant species included, Leersia hexandra Swartz (36.84\%), Aeschynomene fluitans Peter (31.58\%), Typha capensis Rohrb (15.79), Vossia cuspidata Griff (15.0\%), Acroceras macrum Stapf (10.35\%), and Imperata cylindrica (L.) Beauv (5.26\%) (Table 1).

\subsection{Wet and Dry Terrestrial Plant Communities}

\subsubsection{Short Termitaria Habitat}

The commonest plant species in this vegetation zone included; Acroceras macrum Stapf (31.3\%), Aeschynomene fluitans Peter (20.0\%), Ipomea aquatic Forsk (20.0\%), Phragmites australis (Cav.) Trin. ex Steud (12.5\%), Vossia cuspiidata Griff (5\%), Sasbania spp. (5\%), Leersia hexandra Swartz (3.8\%), Amaranthus spp. (1.3\%) and Polygonum senegalense Meisn (1.3\%) (Table 1 and Figure 5). Other plant species which were prevalent included; Hibiscus spp., Heliotropium spp., Paspalum spp. and Setaria ancept Stapf ex Massey. 


\subsubsection{Tall Termitaria Habitat}

This plant community was rarely covered by swamp floods. However, because the zone was characterised by very high water table, woody species were only found on Termitaria. Some of these woody species included; Piliostigma thorningii (Schum.) Milne-Redh., Phyllanthus reticulatus Schum et. Th and Securinega virosa (Roxb. ex Willd.) Pax et $\mathrm{K}$. Hoffm. Although there was no clear dominance among plant species, common plant (grass) species in this plant community were Panicum maximum (26.0\%), Digitaria milanjiana (Rendle) Stapf (15.25\%), Setaria ancept Stapf ex Massey (13.00\%), Cymbopogon validus Stapfex Burtt-Davy (13.0\%, Brachiaria brizantha (Hoscst ex A.Rich.) Stapf (10.92\%, Hibiscus L (10.92\%) and Sorghum verticilliflorum Bremek (10.92\%) (Table 1).

\subsection{Dry Land Plant Community Structure}

Woody vegetation covered most of the northern, southern and eastern parts of Lukanga Swamp, and this vegetation was distinctly recognized as Brachystegia woodland and Acacia-combretum vegetation types.

\subsubsection{Acacia-Combretum Vegetation}

Acacia-Combretum plant community was distributed along the edges of the swamp as well as along streams and the area between Kafue River and the swamp. The results of this plant community are presented in Table 2 and Figure 6. Based on importance value (IV), the most important woody plants were, Combretum ghasalense Engl. et Diels (62.88) followed by Julbernardia paniculata Tropin (27.89) and Bauhinia petersiana Bolle (20.25), while Brachystegia spiciformis Benth (16.66), Acacia xanthophloea Benth (14.34), Acacia polyacantha Wild (12.17) Lannea stuhlmannii (Engl.) Engl. (12.66 and Parinari curatellifolia Planch. ex Benth (12.48) showed co-dominance of importance (Table 2). Relative density of woody species revealed only four plant species as being relatively common, Combretum ghasalense Engl. et Diels (31.25\%), Bauhinia petersiana Bolle (10.0\%), Acacia xanthophloea Benth (6.25\%) while Acacia polyacantha Wild, Parinari curatellifolia Planch. ex Benth were equally common (5.0\%) as nearly all species sampled gave similar values (Table 2). Similarly, results based on percent relative dominance showed Julbernardia paniculata Tropin (25.39\%), Diospyros kirkii Hiern (19.66) and Brachystegia spiciformis Benth (14.16) to be relatively more dominant in this vegetation type.

\subsubsection{Miombo (Brachystegia) Woodland Vegetation}

The results of this vegetation are presented in Tables 3-5. The woody plant structure at Waya habour on the eastern part of the Lukanga Swamp was described by Importance Values given in Table 3 and Figure 7. Plant species dominant in this vegetation type included, Brachystegia woodland Pseudolachnostylis maprouneifolia Pax (90.48), Julbernardia paniculata Tropin (42.30), Albizia adianthifolia (Schmacher) W.F.Wight (33.25), Diplorhynchus condylocarpon (Mull-Arg) Pichon (30.94) and Craterosiphon quarrei Staner (30.18). 
Table 2. Acacia-Combretum woodland vegetation structure at Waya based on species relative frequency and importance values.

\begin{tabular}{|c|c|c|c|c|}
\hline Species & Relative frequency & Relative dominance & Relative density & Importance value \\
\hline Acacia nilotica (L.) Wild. ex Del. & 1.25 & 4.59 & 1.25 & 7.09 \\
\hline Acacia polyacantha Wild & 5.00 & 2.67 & 5.00 & 12.67 \\
\hline Acacia sieberana DC. & 2.50 & 0.08 & 2.50 & 5.08 \\
\hline Acacia xanthophloea Benth & 6.25 & 1.84 & 6.25 & 14.34 \\
\hline Albizia adianthifolia (Schmacher) W.F. Wight & 1.25 & 4.20 & 1.25 & 6.70 \\
\hline Albizia amara (Roxb.) Beauv. & 1.25 & 0.23 & 1.25 & 2.72 \\
\hline Albizia harveyi Fourn. & 1.25 & 0.08 & 1.25 & 2.59 \\
\hline Antidesma venosum (E. Mey. ex Tul.) & 1.25 & 0.00 & 1.25 & 2.50 \\
\hline Bauhinia petersiana Bolle & 10.00 & 0.25 & 10.00 & 20.25 \\
\hline Brachystegia spiciformis Benth. & 1.25 & 14.16 & 1.25 & 16.66 \\
\hline Combretum ghasalense Engl. et Diels & 31.25 & 0.38 & 31.25 & 62.88 \\
\hline Combretum mechowianum O.Hoffim & 1.25 & 2.81 & 1.25 & 5.31 \\
\hline Commiphora mollis (Oliv.) Engl. & 2.50 & 1.57 & 2.50 & 6.57 \\
\hline Diospyros kirkii Hiern. & 1.25 & 19.66 & 1.25 & 22.16 \\
\hline Diplorhynchus condylocarpon (Mull-Arg) Pichon & 1.25 & 3.83 & 1.25 & 6.33 \\
\hline Flacourtia indica (Burm. f.) Merr. & 2.50 & 0.05 & 2.50 & 5.05 \\
\hline Julbernardia paniulata Tropin & 1.25 & 25.39 & 1.25 & 27.89 \\
\hline Lannea stuhlmannii (Engl.) Engl. & 5.00 & 2.66 & 5.00 & 12.66 \\
\hline Lonchocarpus capassa Rolfe & 1.25 & 0.08 & 1.25 & 2.58 \\
\hline Parinari curatellifolia Planch. ex Benth & 5.00 & 2.48 & 5.00 & 12.48 \\
\hline Pericopsis angolensis (Bak.) Ban M & 1.25 & 6.37 & 1.25 & 17.59 \\
\hline Piliostigma thonningii (Schum.) Milne-Redh. & 1.25 & 0.47 & 1.25 & 2.97 \\
\hline Pseudolachnostylis maprouneifolia Pax & 1.25 & 0.08 & 1.25 & 2.58 \\
\hline Schrebera trichoclada Welw. & 1.25 & 0.04 & 1.25 & 2.54 \\
\hline Securineya virosa (Roxb. ex. Willd) Baillon & 1.25 & 0.09 & 1.25 & 2.59 \\
\hline Steganotaenia araliacea Hochst. & 2.50 & 0.03 & 2.50 & 5.03 \\
\hline Terminalia stenostachya Engl. \& Diels & 1.25 & 3.47 & 1.25 & 5.97 \\
\hline \multirow[t]{2}{*}{ Ziziphus mucronata Willd. } & 1.25 & 0.40 & 1.25 & 2.90 \\
\hline & 100 & 100 & 100 & 300 \\
\hline
\end{tabular}

In Maunde forest, dominant species were; Isoberlinia angolensis Hyle and Brenan (87.25) and Julbernardia globiflora Troupin (48.37) (Table 4 and Figure 8). On the northern part of Chilwa Island Albizia adianthifolia (Schmacher) W.F.Wight (135.63), Combretum celastroides Welw. ex C Laws (83.80) and Markamia obtusifolia (Bak.) Sprague (65.53) were the most important (Table 5), while on the southern part of the Island, the woody plant structure was characterized by Albizia adianthifolia (Schmacher) W.F.Wight (109.51), Strychnos pungens Solereder (70.05), Commiphora mollis (Oliver) Engl. (53.00), Diospyros senensis Klotzsch (34.48) and Schrebera trichoclada Welw (19.59) as the most important woody plant species (Table 5 and Figure 9). 


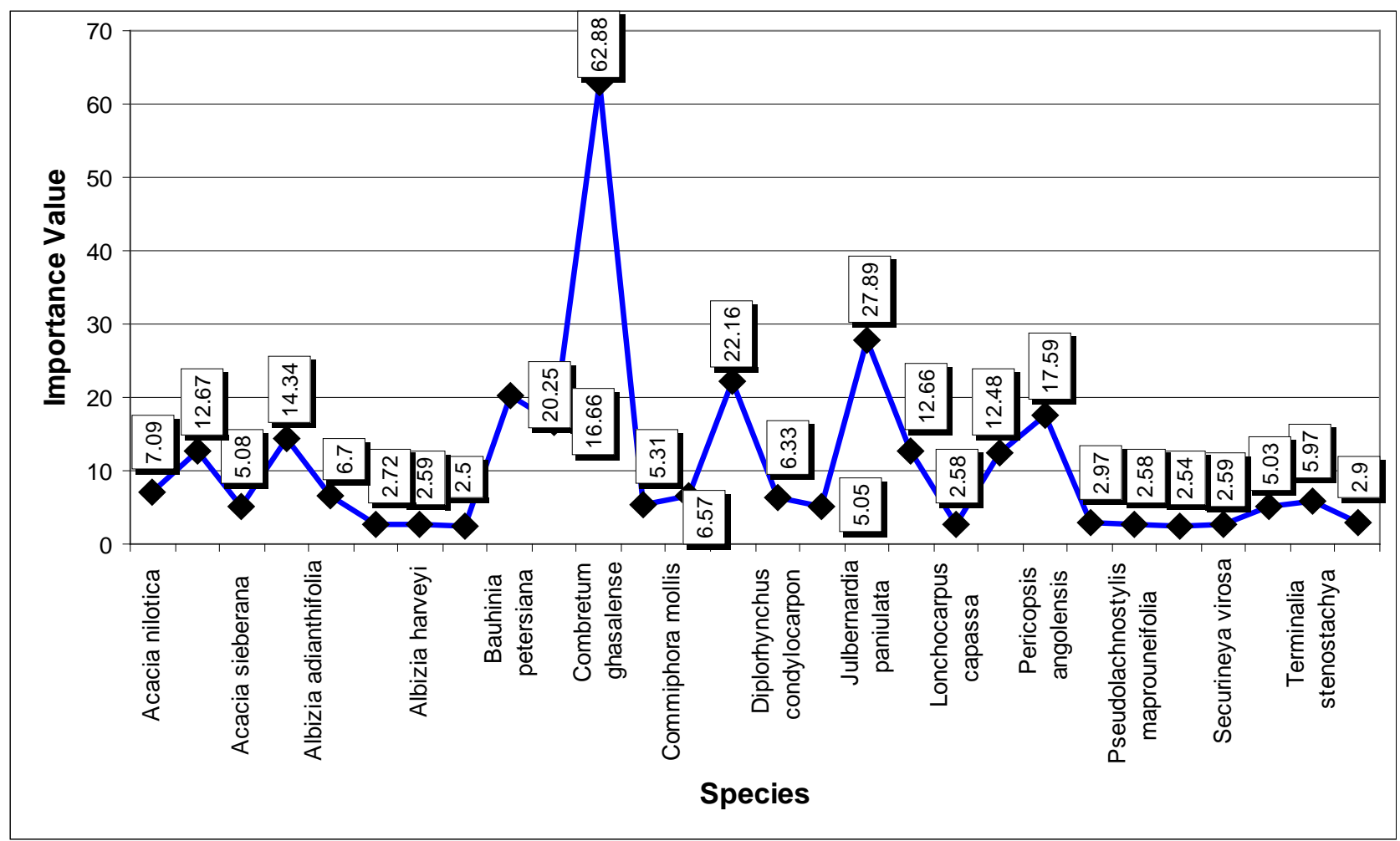

Figure 6. Species importance value in Acacia-Combretum woodland vegetation, Lukanga Ramsar site, Zambia.

Table 3. Miombo woody vegetation structure at Waya East based on species importance values.

\begin{tabular}{ccccc}
\hline SPECIES & Relative Frequency & Relative Dominance & Relative Density & Importance Values \\
\hline Diplorhynchus condylocarpon (Mull-Arg) Pichon & 14.58 & 1.77 & 14.58 & 30.94 \\
Olax obusifolia De Wild & 4.17 & 0.42 & 4.17 & 8.74 \\
Faurea saligna Harvey & 2.08 & 1.63 & 2.08 & 5.80 \\
Craterosiphon quarrei Staner & 14.58 & 1.01 & 14.58 & 30.18 \\
Diospyros kirkii Hiern. & 2.08 & 0.68 & 2.08 & 4.84 \\
Pseudolachnostylis maprouneifolia Pax & 4.17 & 82.15 & 4.17 & 40.48 \\
Julbernardia paniculata Tropin & 20.83 & 0.64 & 20.83 & 42.30 \\
Uapaca nitida Mull. Arg. & 2.08 & 0.43 & 2.08 & 4.60 \\
Albizia adianthifolia (Schmacher) W.F. Wight & 14.58 & 4.08 & 14.58 & 33.25 \\
Combretum ghasalense Engl. et Diels & 4.17 & 3.72 & 4.17 & 12.06 \\
Acacia seyal Del. var. fistula (Schweinf \& Oliv.) & 6.25 & 0.68 & 6.25 & 13.18 \\
Lannea discolor (Sond.) Engl & 2.08 & 0.63 & 2.08 & 4.80 \\
Uapaca kirkiana Mull. Arg. & 2.08 & 0.18 & 2.08 & 4.35 \\
Brachystegia boehmii Taub. & 4.17 & 0.91 & 4.17 & 9.24 \\
Terminalia sericea Burch. ex DC. & 2.08 & 1.06 & 100 & 5.23
\end{tabular}

\section{Discussion}

The use of vegetation communities in wetland assessment protocols for classification and prediction of the condition of a wetland has been well documented 
Table 4. Miombo woodland vegetation structure at Maunde Forest based on species importance values.

\begin{tabular}{ccccc}
\hline SPECIES & Relative Frequency & Relative Dominance & Relative Density & Importance Value \\
\hline Albizia adianthifolia (Schmacher) W.F.Wight & 2.08 & 1.27 & 2.08 & 5.43 \\
Baikiaea africana Hook & 2.08 & 8.42 & 2.08 & 12.59 \\
Brachystegia boehmii Taub. & 4.17 & 7.82 & 4.17 & 16.15 \\
Brachystegia taxifolia Harms & 10.42 & 4.32 & 10.42 & 25.15 \\
Bridelia micrantha (Hochst) Baillon & 2.08 & 1.12 & 2.08 & 5.29 \\
Diospyros batokana Hiern & 4.17 & 4.58 & 4.17 & 12.91 \\
Diplorhynchus condylocarpon (Mull-Arg) Pichon & 4.17 & 3.20 & 4.17 & 11.53 \\
Erythrophleum africanum Harms & 2.08 & 3.44 & 2.08 & 7.61 \\
Isoberlinia angolensis Hyle and Brenan & 37.50 & 12.25 & 37.50 & 87.25 \\
Isoberlinia angolensis Hyle and Brenan & 2.08 & 5.19 & 2.08 & 9.36 \\
Julbernardia globiflora Troupin & 16.67 & 15.03 & 16.67 & 48.37 \\
Julbernardia paniculata Troupin & 4.17 & 3.79 & 4.17 & 12.13 \\
Parinari curatellifolia Planch. ex Benth & 2.08 & 6.60 & 2.08 & 10.77 \\
Pericopsis angolensis Harms & 2.08 & 11.42 & 1.42 & 2.08 \\
Pterocarpus angolensis DC. & 2.08 & 10.11 & 2.08 & 2.08 \\
Bobgunia (Swartzia) Schreb & 2.08 & & 5.59
\end{tabular}

Table 5. Woody vegetation structure at Chilwa Island based on species relative frequency, relative dominance, relative density and importance values.

\begin{tabular}{|c|c|c|c|c|c|}
\hline AREA & SPECIES & Relative Frequency & Relative Dominance & Relative Density & Importance Value \\
\hline & Albizia adianthifolia (Schmacher) W.F.Wight & 31.25 & 73.13 & 31.25 & 135.63 \\
\hline & Azanza garckeana (F. Hoffm.) Exell ex Hillc. & 4.17 & 6.70 & 4.17 & 15.03 \\
\hline \multirow[t]{3}{*}{ Chilwa North } & Combretum celastroides Welw. ex C Laws. & 37.50 & 8.80 & 37.5 & 83.80 \\
\hline & Markamia obtusifolia (Bak.) Sprague & 27.08 & 11.36 & 27.08 & 65.53 \\
\hline & & 100 & 100 & 100 & 300 \\
\hline \multirow[t]{7}{*}{ Chilwa South } & Albizia adianthifolia (Schmacher) W.F.Wight & 27.08 & 55.34 & 27.08 & 109.51 \\
\hline & Boscia salicifolia Oliv & 2.08 & 9.20 & 2.08 & 13.37 \\
\hline & Commiphora mollis (Oliver) Engl & 22.92 & 7.17 & 22.92 & 53.00 \\
\hline & Diospyros senensis Klotzsch & 16.67 & 1.15 & 16.67 & 34.48 \\
\hline & Schrebera trichoclada Welw & 4.17 & 11.25 & 4.17 & 19.59 \\
\hline & Strychnos pungens Solereder. & 27.08 & 15.88 & 27.08 & 70.05 \\
\hline & & 100 & 100 & 100 & 300 \\
\hline
\end{tabular}

[24] [25] [26] and much research has been devoted to this subject [27] [28] [29]

[30]. Researchers have listed many other advantages in using plant species to assess vegetation structure and wetland condition; firstly, plants are immobile and have very high diversity, and are very sensitive to environmental stressors such as hydrological alteration, eutrophication or nutrient enrichment, sediment loading and many other pollutants [31] [32] [33]. Secondly, the vast knowledge in taxonomy, methodologies and techniques has enabled researchers to make meaningful predictions about the condition of many wetlands under consideration 


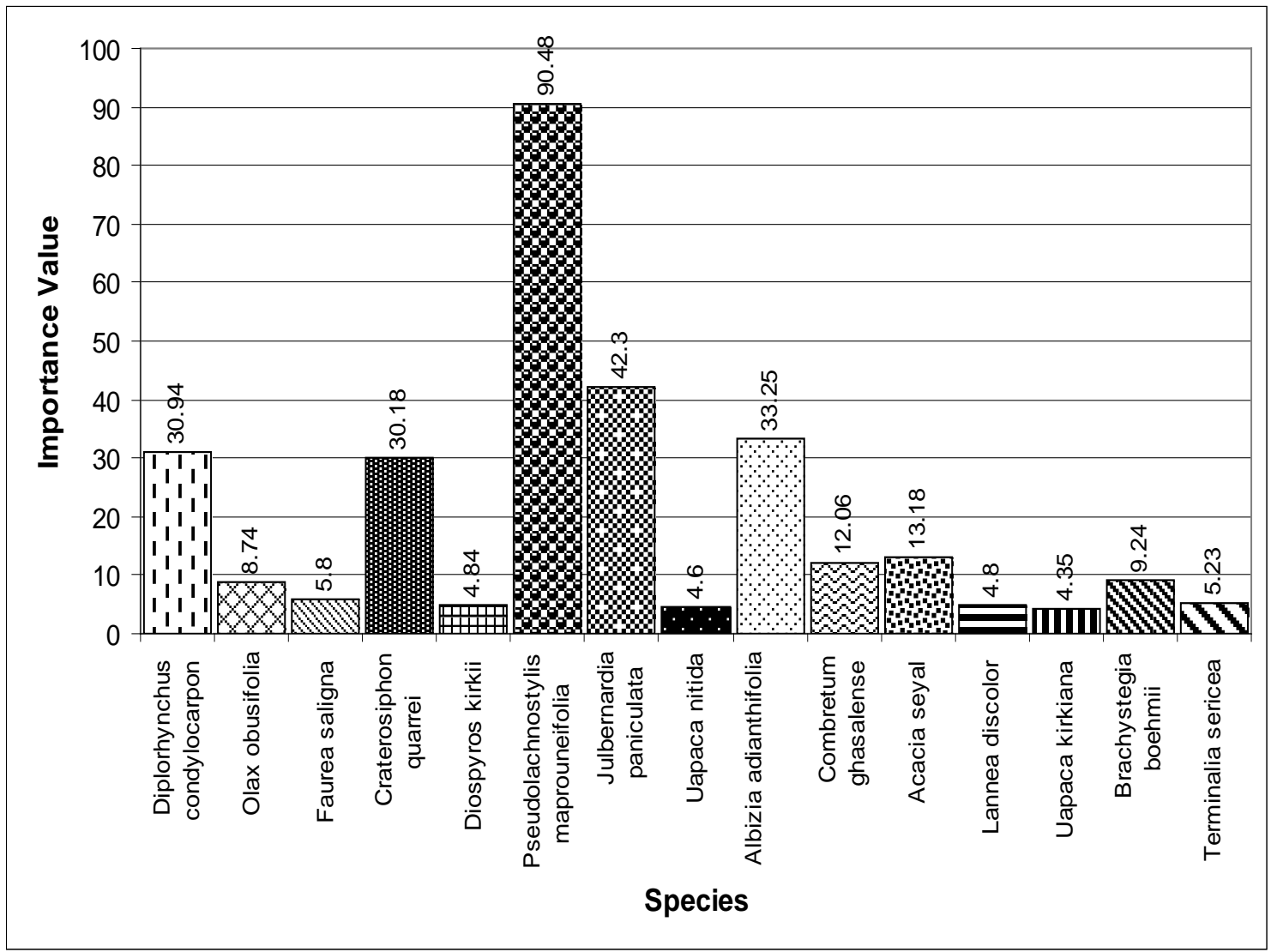

Figure 7. Species importance value in Miombo Woody vegetation structure at Waya East.

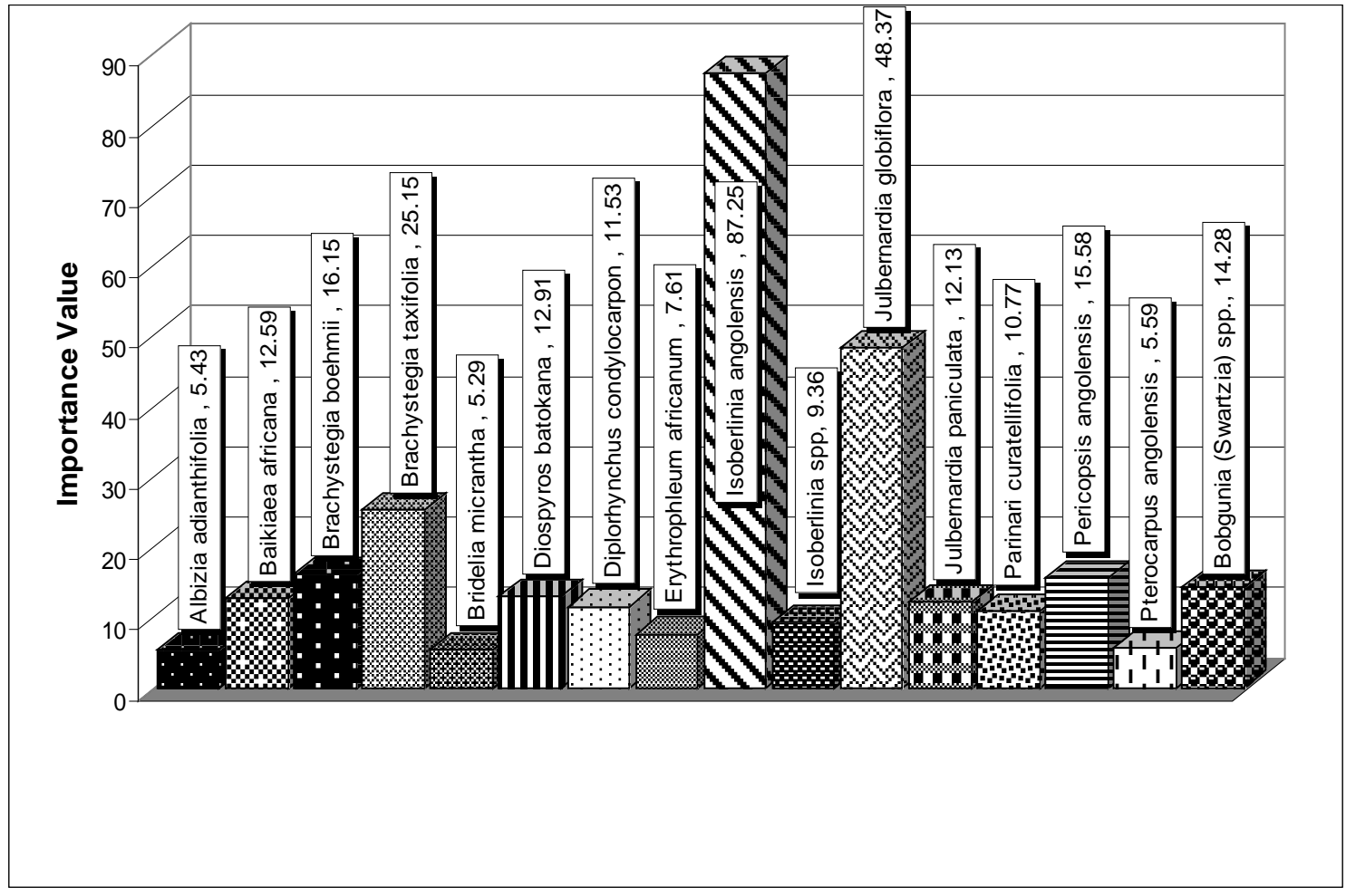

Figure 8. Species importance value Miombo Woodland vegetation structure at Maunde Forest, Lukanga Ramsar site, Zambia. 


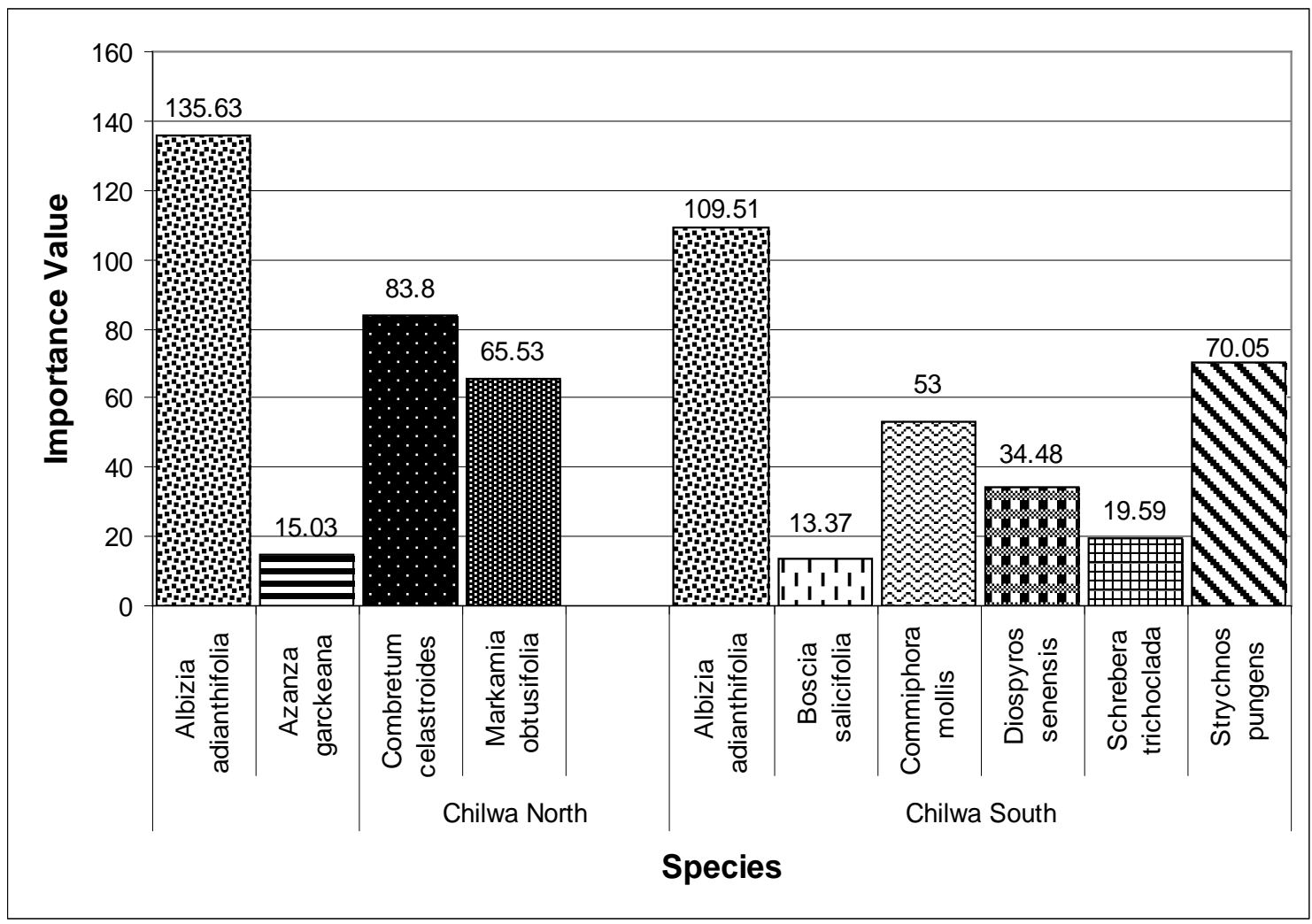

Figure 9. Species importance value in woody vegetation structure at Chilwa Island, Lukanga Ramsar site, Zambia.

[33] and thirdly, the number of plant taxa growing in wetlands is large and offers numerous potential attributes for method development [31]. However, we took note of the limitations that might arise in using this approach because we did not take into account an inclusion of the assessment of rooted submerging and floating macrophytes to give a comprehensive structure of the Lukanga wetland as earlier recorded in other studies [33]. We therefore, strictly relied on emergent macrophytes as indicators to describe the structure and to classify the Lukanga wetland.

In determining the hierarchical classification of wetlands we on the basis of our findings, viewed the Lukanga wetland as a littoral swamp which had developed the ecological character of a dambo palustrine wetland system. Furthermore, the species composition of plant hydrophytes in this wetland signified a dambo structure. A dambo is known to be a seasonally waterlogged palustrine bottomland wetland associated with the headwaters of river systems draining the plateau areas, and is usually covered with grassland plant species and aquatic macrophytes mainly sedges [34] [35].

\subsection{Aquatic Macrophytes Structure}

Zonation patterns of macrophytes has been well documented in various studies [33] [36] [37] and is usually developed in succession zones between the woodland and deep water habitats or up to the river channel, each zone being dominated by specific plant species. It was also earlier shown [38] that macrophyte 
composition and distribution varies with hydrology, substrate type and nutrient availability [39] as well as other factors such as land use and sediments. These factors and human activities such as moving of fishing crafts (Figure 10) seem to have a profound influence on the distribution of plants in Lukanga Swamp.

Plant dominance [40] such as the dominance of Phragmites australis (Cav.) Trin. ex Steud, Typha capensis Rohrb, Aeschynomene fluitans Peter, Polygonum senegalense Meisn as obtained in this study showed and clearly suggests a condition of a largely stressed wetland habitat. This is because these plant species are generally considered as indicators of silted, muddy and shallow water environment, and are usually well established in such areas and to some extent may be prolific and grow readily even in excessively eutrophic and polluted environments as also noted in other similar studies [32] [38] [41]. In particular, Phragmites australis (Cav.) Trin. ex Steud ( $P$. communis trinius) is an invasive and successful plant, and has a wide distribution in the world. The plant disperses through seed, cut fragments of stem or rhizomes and seed spreads by wind, and because of this ability the plant is readily established in shallow and muddy parts of many wetlands [42]. Similarly, Typha capensis Rohrb is a well-known indicator of a wetland habitat and the species prefers not only muddy, silted and stagnant water habitats, but it is successful in wet soils with high content of organic matter [43] [44] [45]. Typha capensis Rohrb and its congeneric species reproduce

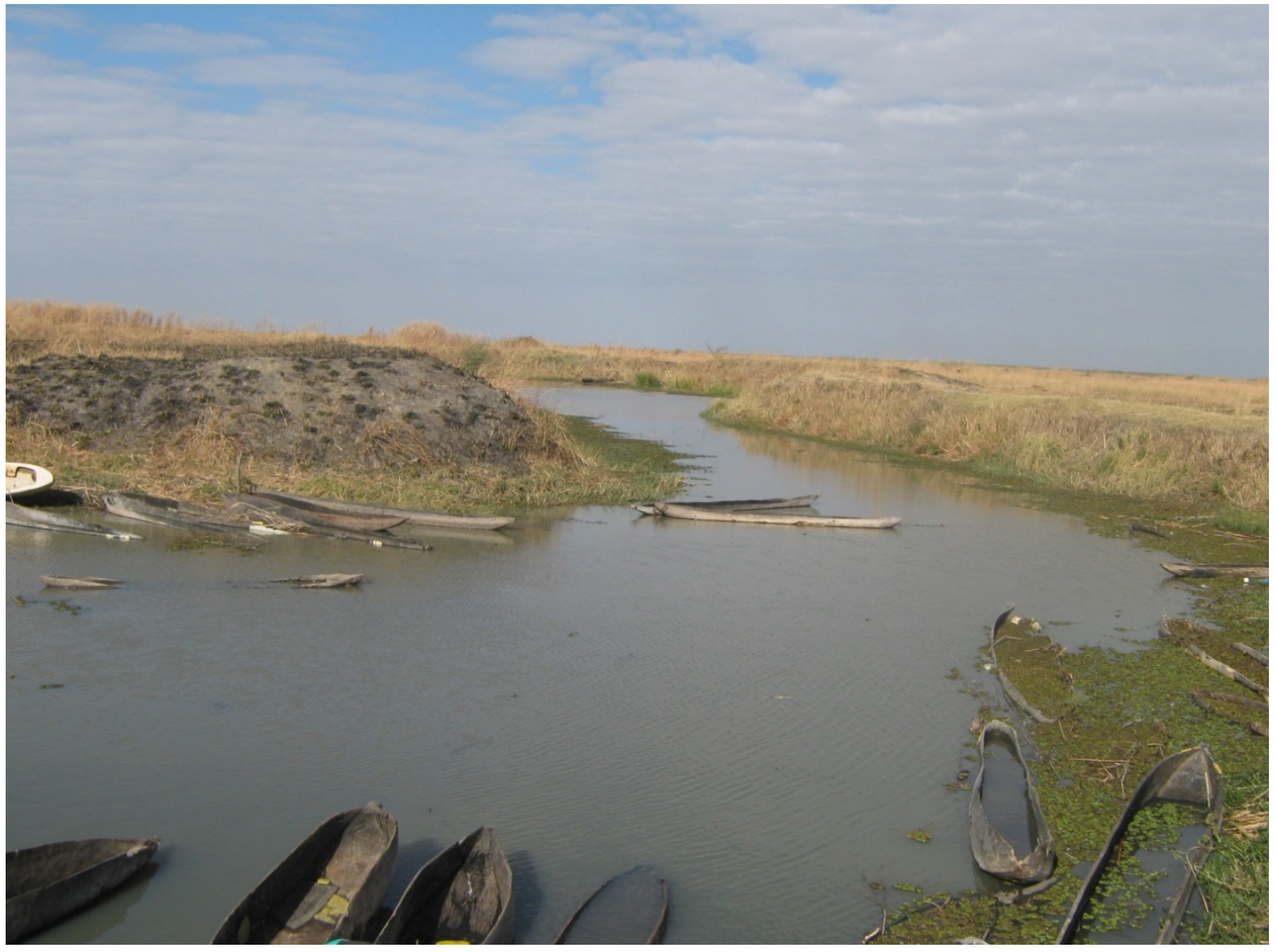

Figure 10. Water vessels as plant dispersal gents and distribution on the swamp. 
very rapidly through rhizomes and seeds [43] [46] and its dominance on the eastern side of the swamp could indicate occurrence of heavy sedimentation due to deforestation partly for firewood used to dry fish (see Figure 1(b)).

In contrast, Nymphaea capensis Thunb is an aquatic rhizomatous perennial emergent herbaceous plant and it grows in many types of stagnant water bodies, while Vossia cuspidata Griff and Cyperus papyrus L. establish communities in stable flowing water environments [44]. Although we did not carry out assessment of Cyperus papyrus and Vossia cuspidata communities, these have limited distribution in this wetland for a number of reasons. Earlier findings [44] [47] revealed some of the factors causing decline of Cyperus papyrus L. in the Nile River in Sudan and Egypt, and among the reasons discussed were high amplitudes between the dry and flood periods. Ordinarily, Cyperus papyrus L. is more typical of stable hydrological environment and may tolerate low $\mathrm{pH}$, but a papyrus swamp cannot endure unstable water flows as also noted elsewhere [47] because of the plant's poor rooting system in which the plant may anchor itself in soil during the dry season and during high floods, it may die due to drowning. Much of the papyrus swamp in this wetland has since been replaced by either Vossia cuspidata Griff or by Phragmites australis (Cav.) Trin. ex Steud.

\subsection{Termitaria Habitat Structure}

Whereas factors influencing the distribution of termite mounds have not been fully investigated and understood, the occurrence of Termitaria in Lukanga Swamps seems to be a result of soil type, soil moisture and hydrology. Accordingly, tall Termitaria are built by two species of termites, the Macroterms spp. and Odontoterms spp. and that these build large mounds from subsoil in a variety of soil types. Earlier records [27] [48], provide good descriptions of floristic composition of Termitaria habitats. Our results confirm the species of plants usually occurring on these mounds, and these were mostly woody and herbaceous plants species. In general, tall Termitaria displayed high diversity of plant species characteristically different from the surrounding landscape because they provided excellent conditions for plant growth. In similar studies [47] it was shown that termitaria had very high content of nutrients such as carbon, nitrogen, calcium, potassium, magnesium, clay/silt and were alkaline as well as having very high content of moisture and rich in humus. Consequently, these habitants are widely cultivated in Lukanga Swamp.

Short Termitaria habitat was a significant feature in Lukanga Swamp, and this is recognized by the dotted grey or dark short mounds associated with herbaceous plant species. Short Terminaria occurs throughout Zambia in dambos, swamps and flood plains. These mounds are built by Cubitermes spp. of termites, and are built under conditions induced by impermeable nature of clay soils resulting from extreme hydrological conditions of severe drought in dry season and water logging in wet season. Short Termitaria habitat clearly defines the transitional nature of the environment between the terrestrial and aquatic systems. This habitant was noticeably diverse in herbaceous species including 
the prevalence of Setaria spp., Hibiscus spp., Heliotropium spp. and Vernomia spp. Kirchmair et al. (2012) [27] [28]. Because of its structure and the transitional nature of periodic dryness and wetness, the area remains an important habitat for conservation.

\subsection{Woody Vegetation Structure}

Our findings of the assessment of woody vegetation at Chilwa Island and the eastern part of Lukanga Swamp (Table 2, Table 3, and Table 5) show the dominance of the under storey plant species as opposed to Maunde protected forest which had dominance of the upper storey (Table 4). The high presence and the dominance of Pseudolachnostylis maprouneifolia Pax, Diplorhynchus condylocarpon (Mull-Arg) Pichon, Craterosiphon quarrei Staner, Strychnos pungens Solereder. Commiphora mollis (Oliver) Engl, Combretum ghasalense Engl. et Diels, Combretum celastroides Welw. ex C Laws, Bauhinia petersiana Bolle and Markamia obtusifolia (Bak.) Sprague, strongly suggests the excessive tree removal of the upper storey canopy plant species.

The structure of Miombo vegetation has already been widely reported in various studies [49] [50] and invariably these plant species seem to represent the under canopy cover. Therefore, the current findings strongly suggest deforestation to be the probable cause of excessive removal of upper storey plant species and this is generally a result of increasing agriculture, charcoal production and fuel wood collections which are important means of livelihood on the eastern part of the Lukanga Swamp.

\section{Conclusion and Recommendations}

\subsection{Conclusion}

This study highlighted the species composition and structure of the Lukanga Ramsar site, attendant potential threats on conservation of wetland biodiversity and the need for concerted effort to sustain the ecological functioning of the Lukanga wetland and the need for further investigations.

\subsection{Recommendations}

On the basis of our findings and the issues raised in this study, we propose future investigations to include the following:

Wise use of Lukanga Swamp: "Wise use of wetlands is the maintenance of their ecological character, achieved through the implementation of ecosystem approaches, within the context of sustainable development'. (RAMSAR, 2010). The Lukanga Swamp is among the most significantly important wetlands in Zambia. The significance lies in the number of species of animals which have been recorded as potentially present in the area. About 127 species of mammals, 316 species of birds, 69 species of reptiles and 27 species of amphibians may potentially occur in the Lukanga Swamp region [15] and it is also an important fishery (see Figure 1(d)). Recent records have revealed that Lukanga Swamp wetland produces in excess of 1710 tons of fish annually [51] [52]. 
Nevertheless, while this may be the case, wise use of this wetland is threatened by increasing pressure resulting from over exploitation, deforestation and expanding agricultural activities including grazing (see Figure 1(a)).

Although the aspect of unsustainable utilization of resources was not covered in the present study, our view emphasizes the depletion of fish and wildlife populations and as such we recommend that detailed investigations be carried out to confirm and substantiate these concerns, and to bring out findings that may provide guidance for the conservation plans.

Wetland condition of Lukanga Swamp: Although this aspect was also not investigated in the current study, understanding wetland health is essential in the effective management of and monitoring of a wetland. Wetland condition refers to the health of an ecosystem, and it includes the stability and sustainability of the ecosystem to withstand environmental stress [5] and it further describes the condition as an ecosystem having the capacity to support a diverse community of organisms and perform functions compared to that of a local unimpaired site [6]. We recommend that a study to determine wetland condition of the Lukanga Swamp should be carried out.

Environment flows and wetland recharge functions: Knowledge of environmental flows is important to assist in the management of this wetland ecosystem. An environmental flow is understood to be the quality, quantity and timing or water flows required to sustain freshwater ecosystem and the human livelihoods and wellbeing that depend on these ecosystems [53] [54] [55] [56] and it is also important to note that there are other water requirements such as the ecological flow needs which may refer to the flow levels required in a water body for flora and fauna and habitat processes present within that water body and its margins [54]. There are several reasons for proposing assessment of environmental and ecological flows for Lukanga Swamp. Firstly, the watershed of Lukanga Swamp is largely limited and streams in this watershed are relatively intermittent and may flow only for three to four months in a year. This limitation coupled with excessive reduction of forests in the surrounding area is inevitably a serious threat as volume of inflows may remain correspondingly low to feed the swamp. Considerable amounts of inflow in the swamp come from the Kafue River as an overland flow. However, this view would require detailed investigations. Secondly, the relationship between water and forests has been well investigated and comprehensively discussed elsewhere [57] [58] [59], and it should be well understood that the vegetation around the Lukanga Swamp is an integral part of the wetlands landscape. Studies have shown that forests play a significant role in the interactions between ground water of wetland ecosystem through a recharge function [57] as well as controlling flood flows, water quality and erosion. Our view is that it would be important that research should be carried out to determine the significance of this function. Thirdly, a wetland carries an important function of remediating various nutrients and sediments [39] [60] [61] [62]. Unlike the riverine (flood plain) wetlands, the Lukanga Swamp is a depression and almost entirely closed wetland. This kind of limitation has been 
discussed [61] and our view therefore is that with this limitation in mind, it is highly probable that the Lukanga Swamp cannot effectively sequester excess nutrients, sediments and other pollutants compared to a flood plain wetland. This inability may lead to increased eutrophication and reduced fisheries habitat quality.

Wetland fires: Whether or not fires cause changes in the structure of vegetation has been a subject of investigation and debate for a long time. While this was not investigated in this study, fires occur annually in many parts of Zambia during the dry season. Numerous and well-documented studies have been done on the effects of fires on wetlands [40] [58] [59] [63] [64] [65]. These studies have revealed that although wetland fires are an integral part of wetland landscape, their effects remain less understood. One of our hypotheses on this subject nevertheless suggests that although fires may remove much of the vegetation in a wetland, they can not only change the structure and configuration of a wetland, but that the removal of organic soils and change in water chemistry may have a negative effect on other organisms. We believe that research on this aspect should be pursued in Lukanga Swamp. Furthermore, potential effects of fires on woody vegetation around Lukanga Swamp should be viewed with some serious consideration as studies elsewhere [63] [64] [65] [66] [67] tend to suggest that while fires have values for reshaping the ecological landscape, they are seemingly detrimental in other respects. Our recommendation for fire research in Lukanga is based on the argument that excessive vegetation removal in this area may have serious hydrological implication on this wetland particularly in the recharge function. Such consequences include not only severe reduction in subsurface and underground water recharge, but increased erosion and decline in water quantity for the wetland which must be thoroughly investigated.

\section{References}

[1] Ramsar Convention Secretariat (2010) Wetland inventory: A Ramsar Framework for Wetland Inventory and Ecological Character Description. Ramsar Handbooks for the Wise Use of Wetlands. 4th Edition, Ramsar Convention Secretariat, Gland.

[2] Perera, N.P. (1982) The Ecology of Wetlands (Dambos) of Zambia, and Their Evaluation for Agriculture-A Model for the Management of Wetlands in Sub-Humid Eastern and Southern Africa. International Journal of Ecology and Environmental Science, 8, 27-38.

[3] Breen, C.M., Quinn, N.W. and Mander, J.J. (1997) Wetland Conservation and Management in Southern Africa: Challenges and Opportunities. Summary of the SADC Wetlands Conservation Survey Reports. City Printing Works Limited, Harare.

[4] Wolt, K.L., Noe, G.B. and Ahn, C. (2013) Hydrologic Connectivity to Streams Increases Nitrogen and Phosphorus Inputs and Cycling in Soils of Created and Natural Floodplain Wetlands. Journal of Environmental Quality, 42, 1245-1255. https://doi.org/10.2134/jeq2012.0466

[5] Faber-Langendoen, D., Hedge, C., Kost, M., Thomas, S., Smart, L., Smyth, R., Drake, J. and Menard, S. (2012a) Assessment of Wetland Ecosystem Condition across Landscape Regions: A Multi-Metric Approach. Part A. Ecological Integrity 
Assessment Overview and Field Study in Michigan and Indiana. U.S. Environmental Protection Agency Office of Research and Development, Washington, DC, 138 p.

[6] Faber-Langendoen, D., Rocchio, J., Thomas, S., Kost, M., Hedge, C., Nichols, B., Walz, K., Kittel, G., Menard, S., Drake, J. and Muldavin, E. (2012b) Assessment of Wetland Ecosystem Condition across Landscape Regions: A Multi-Metric Approach. Part B. Ecological Integrity Assessment Protocols for Rapid Field Methods (L2). U.S. Environmental Protection Agency Office of Research and Development, Washington DC, $111 \mathrm{p}$.

[7] Hilty, J. and Merenlender, A. (2000) Faunal Indicator Taxa Selection for Monitoring Ecosystem Health. Biological Conservation, 92, 185-197. https://doi.org/10.1016/S0006-3207(99)00052-X

[8] Zambia Wildlife Authority (2005) Ministry of Tourism, Environment and Natural Resources. National Wetlands Report, Zambia Wildlife Authority, Chilanga.

[9] McCartney, M. (2007) Technical Note: Hydrology of the Lukanga Swamp, Zambia. Technical Note, International Water Management Institute (IWMI), Colombo.

[10] King, L. (1978) The Geomorphology of Central and Southern Africa. In: Werger, M.J.A., Ed., Biogeography and Ecology of Southern African, Dr. W. Junk Publishers, Hague, 1-17. https://doi.org/10.1007/978-94-009-9951-0 1

[11] Remond, W.A. (2009) Gondwanaland: In Microsoft Encarta, Microsoft Corporation.

http://www.windows.umic.edu/cgi-bin/tourcgi?link=/earth/interior/plate tectonics $\underline{\mathrm{html} \& \mathrm{sw}=\mathrm{f}}$

[12] Encyclopaedia Britannica (2010) Gondwana. Encyclopaedia Britannica, Ultimate Reference Suite, Chicago. http://www.britannica.com/place/Gondwana

[13] Trapnell, C.G. and Clothier, J.N. (1996) The Soils, Vegetation and Traditional Agriculture of Zambia. Vol. I. Central and Western Zambia Ecological Survey (1932-1936). Red Cliff Press Ltd., Bristol, 96 p.

[14] Sharma, T.C. (1985) Stochastic Characteristics of Rainfall-Runoff Processes in Zambia. Hydrological Science Journal, 30, 497-512.

https://doi.org/10.1080/02626668509491014

[15] Matiza, T. and Chabwela, H.N. (1991) Wetland Conservation Conference for Southern Africa. Proceedings of the Southern African Development Coordinating Conference, Gaborone, 3-5 June 1991.

[16] McCartney, M., Cai, X. and Smakhtin, V. (2013) Evaluating the Flow Regulating Functions of Natural Ecosystems in the Zambezi River Basin. IWMI Research Report 148, International Water Management Institute (IWMI), Sri Lanka, 59 p.

[17] Bingham, M.G., Willemen, A., Wursten, B.T., Ballings, P. and Hyde, M.A. (2017) Flora of Zambia: Location Details: Lukanga Swamps.

http://www.zambiaflora.com/speciesdata/locationdisplay.php?location $\mathrm{id}=110$

[18] Palgrave, K.C. and Drammund, R.B. (1990) Trees of Southern Africa. Struik Publishers, Cape Town.

[19] Verma, P.S. and Agarwal, V.K. (2005) Environmental Biology: Principles of Ecology. S. Chand and Company Limited, New Delhi.

[20] Lucey, T. (2002) Quantitative Techniques. Book Power, London.

[21] Mueller-Dombois, D. and Ellenberg, H. (1974) Aims and Methods of Vegetation Ecology. John Wiley and Sons, Toronto, 547 p.

[22] Campbell, B.M., Cunlite, R.N. and Gambiza, J. (1995) Vegetation Structure and Small Scale Pattern in Miombo Woodland, Marondera, Zimbabwe. Bothlia, 25, 
121-126. https://doi.org/10.4102/abc.v25i1.721

[23] US EPA (2000) Methods for Evaluating Wetland Condition: Using Vegetation to Assess Environmental in Wetlands. Office of Water, U.S. Environmental Protection Agency, Washington DC. https://www.epa.gov/sites/production/files/documents/wetlands 11algae.pdf

[24] Madsen, J.D. (1999) Point Intercept and Line Intercept Methods for Aquatic Plant Management APCRP Technical Notes Collection (TN APCRP-M1-02). Army Engineer Research and Development Center, Vicksburg. http://www.wes.army.mil/el/aqua

[25] Thomaz, S.M. and da gunha, E.R. (2010) The Note of Macrophytes in Habitat Structuring in Aquatic Ecosystems: Methods of Measurements, Causes and Consequences on Animal Assemblages, Composition and Biodiversity. Acta Limnologica Brasiliensia, 22, 218-236. https://doi.org/10.4322/actalb.02202011

[26] Chabwela, H.N. and Siwela, A. (1986) The Vegetative Structure of the Kafue Flats, North Bank, after the Construction of the Dams. Proceedings 7 th Symposium on Aquatic Weeds, Loughborough, 61-72.

[27] Ellenbroek, G.A. (1987) Ecology and Productivity of an Africa Wetland System. The Kafue Flats, Zambia, Dr. W. Junk, Boston, 266 p. https://doi.org/10.1007/978-94-009-4051-2

[28] Thompson, K. (1985) Emergent Plants of Permanent and Seasonally-Flooded Wetlands. In: Denny, P., Ed., The Ecology and Management of African Wetland Vegetation. A Botanic Account of African Swamps and Water Bodies, Dr. W. Junk, Boston.

[29] Denny, P. (1985) Submerged and Floating-Leaved Aquatic Macrophytes (Euhydrophytes). In: Denny, P., Ed., The Ecology and Management of African Wetland Vegetation. A Botanic Account of African Swamps and Water Bodies, Dr. W. Junk, Boston.

[30] U.S. EPA (2008) Methods for Evaluating Wetland Condition: Biogeochemical Indicators. Office of Water, U.S. Environmental Protection Agency, Washington DC.

[31] Backéus, I., Petterson, B., Stromquit, L. and Ruffo, C. (2006) Tree Communities and Structural Dynamic in Miobo (Brachystegia-Julbernardia) Woodland, Tanzania. Forest Ecology and Management, 230, 171-178. https://doi.org/10.1016/j.foreco.2006.04.033

[32] US EPA (2002) Methods for Evaluating Wetland Condition: Using Vegetation to Assess Environmental Conditions in Wetlands. Office of Water, U.S. Environmental Protection Agency, Washington DC Kasimona, V. (1998) Hydrological Evaluation of Lukanga Swamp. Proceedings of the Environmental Council of Zambia, Lusaka.

[33] Abobi, S.M., Ampofo-Yehoah, A., Kpodonu, T.A., Alhassan, E.H., Abarike, E.D., Atindaana, S.A., Akongyuure, D.N., Konadu, V. and Twumasi, F. (2015) SocioEcological Importance of Aquatic Macrophytes to Some Fishing Communities in the Northern Region of Ghana. Bio Diversity_Elixir International Journal, 79, 30432-30437.

[34] Constantin, J., von der, H. and New, M.G. (2003) The Role of a Dambo in the Hydrology of a Catchment and the River Network Downstream. Hydrology and Earth System Science, 7, 339-357. https://doi.org/10.5194/hess-7-339-2003

[35] Fynn, R.W.S., Murray-Hudson, M., Dhliwayo, M. and Scholte, P. (2015) African Wetlands and Their Seasonal Use by Wild and Domestic Herbivores. Wetlands Ecology Management, 23, 559-581. https://doi.org/10.1007/s11273-015-9430-6

[36] Chabwela, H.N. and Ellenbroek, G.A. (1990) The Impact of Hydroelectric Devel- 
opment on the Lechwe and Its Feeding Grounds at Kafue Flats, Zambia. In: Whigham, D.F., Good, R.E. and Kvet, J., Eds., Wetland Ecology and Management: Case Studies, Kluwer Academic Publishers, London. https://doi.org/10.1007/978-94-009-2115-3 11

[37] Gaudet, J.J. (1992) Structure and Function of African Floodplains. Journal of the East Africa Natural History Society and National Museum, 82, 1-32.

[38] Kissoon, L.T., Jacob, D.L., Hanson, M.A., Herwig, B.R., Bowe, S.E. and Otte, M.L. (2013) Macrophytes in Shallow Lakes: Relationships with Water, Sediment and Watershed Characteristics. Aquatic Botany, 109, 39-48. https://doi.org/10.1016/j.aquabot.2013.04.001

[39] Reddy, K.R., DeLaune, R. and Craft, C.B. (2010) Nutrients in Wetlands: Implications to Water Quality under Changing Climatic Conditions. Final Report Submitted to U. Environmental Protection Agency, EPA Contact No. EP-C-0001.

[40] Muraza, M., Mayo, A.W. and Norbert, J. (2013) Wetland Plant Dominance, Density and Biomass in Mara River Basin Wetlands Upstream of Lake Victoria, Tanzania. International Journal of Science and Technology Research, 2, 348-359.

[41] Lansdown, R.V. (2015) Phragmites australis. The IUCN Red List of Threatened Species. e.T164494A79857020.

[42] Tilley, D.J. and St. John, L. (2012) Plant Guide for Common Reed (Phragmites australis) USDA-Natural Resources Conservation Service, Aberdeen, ID Plant Materials Center. 83210-0296. http://plant-materials.nrcs.usda.gov

[43] Mussanjo, E., Kamanga-Thole, G., Mtambo, C. and Chisanga, O. (2014) Evaluation and Natural Regeneration and Trees Species Diversity in Miombo Woodlands in Malawi. Journal of Biodiversity Management Forestry, 3, 3.

[44] Price, C., Gosling, A., Golus, C. and Weslake, M. (2007) Wetland Assessment Techniques Manual for Australian Wetlands. Wetland Care Australia, Ballina.

[45] Sarag, S.M. (2003) Ecology and Biomass Production of Cyprus Papyrus L on the Nile Bank at Damietta, Egypt. Journal of Mediterranean Ecology, 4, 15-24.

[46] Smith, S.G. (1986) The Cattails (Typha): Interspecific Ecological Differences and Problems of Identification. Lake and Reservoir Management, 2, 357-362. https://doi.org/10.1080/07438148609354657

[47] Howard-Williams, C. and Gaudet, J.J. (1985) The Structure and Functioning of African Swamps. In: Denny, P., Ed., The Ecology and Management of African Wetland Vegetation. A Botanic Account of African Swamps and Water Bodies, Dr. E. Junk Publishers, Boston. https://doi.org/10.1007/978-94-009-5504-2 6

[48] Lawton, R.M. (1978) A Study of the Dynamic Ecology of Zambian Vegetation. Journal of Ecology, 66, 175-198. https://doi.org/10.2307/2259187

[49] Muboko, N., Mushonga, M.R., Chibuye, N., Mashapa, C. and Gandiwa, E. (2013) Woody Vegetation Structure and Composition in Mupembe Nature Reserve, Eastern Zimbabwe. Journal of Applied Sciences Environment, 17, 475-481.

[50] Jew, E.K.K., Dougill, A.J., Sallu, S.M., O’Connel, J. and Benton, T.G. (2016) Miombo Woodland under Threat: Consequences for Free Diversity and Carbon Storage. Forest Ecology and Management, 361, 144-153. https://doi.org/10.1016/j.foreco.2015.11.011

[51] Mwabo, L., Valkonen, S. and Kuutti, V. (2008) Structure and Dynamics of Miombo Woodland Stands at Kitulangulo Forest Reserve, Tanzania. Finnish Forest Reserve Institute, 98, 10-19.

[52] Zambia Wildlife Authority (2006) National Report on Wetlands. Ministry of Tourism, Environment and Natural Resources, Chilanga. 
[53] Denny, P. (1985b) The Structure and Functioning of African Euhydrophyte Communities. The Floating Leaved and Submerged Vegetation. In: Denny, P., Ed., The Ecology and Management of African Wetland Vegetation. A Botanic Account of African Swamps and Water Bodies, Dr. W. Junk, Boston.

[54] Denny, P. (1985) Wetland Vegetation and Associated Plant Life Terms. In: Denny, P., Ed., The Ecology and Management of African Wetland Vegetation. A Botanic Account of African Swamps and Water Bodies, Dr. W. Junk, Boston, 1-18.

[55] U.S. EPA (2002) Methods for Evaluating Wetland Condition: Biological Assessment Methods for Birds. Office of Water, U.S. Environmental Protection Agency, Washington DC.

[56] Young, R.J.B. and Marston, F. (2000) Estimating the Water Requirement for Plants of Flood Plains Wetlands: A Guide. Occasional Paper 04/00, Land and Water Resources Research and Development Corporation, Canberra.

http://www.twrrdc.gv.au

[57] Cowardin, L.M., Carter, V., Golet, F.C. and LaRoe, E.T. (1979) Classification of Wetlands and Deep-Water Habitats of the United States. U.S. Fish and Wildlife Service, FWS/OBS-79/31, Washington DC.

[58] WWF. The Miombo Ecoregion; Conservation for Future Generations. WWF Southern Africa Programme Office, Harare.

[59] Campbell, B. (1996) The Miombo in Transition: Woodlands and Welfare in Africa. CIFOR, Bogor.

[60] Timberlake, J.R. (2000) Biodiversity of the Zambezi Basin Wetlands. Consultancy Report for IUCN ROSA, Vol. 4, Biodiversity Foundation for Africa, Bulawayo/Zambezi Society, Harare.

[61] Lue-Mbizvo (2003) Promoting Water Demand Management from Local to Regional Level. Proceedings of the Exchange Visit Seminar for Directors, Francistown, 9-11 July 2003.

[62] Chidumayo, E.N. and Gumbo, D.J. (2010) The Dry Forests and Woodlands of Africa. Earth Scan, London.

[63] Jeffery, R.C.V., Chabwela, H.N., Howard, G. and Dugan, P.J. (1986) Managing the Wetlands of Kafue Flats and Bangweulu Basin. Proceedings of the WWF-Zambia Wetlands Project Workshop, Musungwa Safari Lodge, 5-7 November 1986.

[64] Chenje, M. and Johnson, P. (1994) Status of the Environment in Southern Africa. Penrose Press, Johannesburg.

[65] Bos, E., Muhweezi, A. and West, K. (2005) From Conversion to Conservation: Fifteen Years of Managing Wetlands for People and Environment in Uganda. WID, Kampala and IUCN Eastern Africa Regional Programme Office Nairobi, Kenya.

[66] Quesne, T.L., Mathews, H.J., Von de Heyden, C., Wickel, A.J., Wilby, R., Hartmann, J., Pegram, G., Kistin, E., Blate, G., de Freitas, G.K., Levine, E., Guthrie, C., McSweeney, C. and Sindorf, N. (2010) Freshwater Ecosystem Adaptation to Climate Change in Water Resources Management and Biodiversity Conservation. Water Partnership Programme.

[67] Chirwa, P.W., Syampungani, S. and Geldenhuyd, C.J. (2008) The Ecology and Management of the Miombo Woodlands for Suitable Livelihoods in Southern African: A Case for No-Timber Forest Production. Southern Forests, 70, 237-245. https://doi.org/10.2989/SF.2008.70.3.7.668 
Submit or recommend next manuscript to SCIRP and we will provide best service for you:

Accepting pre-submission inquiries through Email, Facebook, LinkedIn, Twitter, etc. A wide selection of journals (inclusive of 9 subjects, more than 200 journals)

Providing 24-hour high-quality service

User-friendly online submission system

Fair and swift peer-review system

Efficient typesetting and proofreading procedure

Display of the result of downloads and visits, as well as the number of cited articles Maximum dissemination of your research work

Submit your manuscript at: http://papersubmission.scirp.org/

Or contact oje@scirp.org 